\title{
A Flame Particle Tracking Analysis of Turbulence-Chemistry Interaction in Hydrogen-Air Premixed Flames
}

\author{
Harshavardhana A. Uranakara ${ }^{1}$, Swetaprovo Chaudhuri ${ }^{1,2^{*}}$, Himanshu L. Dave ${ }^{1}$, \\ Paul G. Arias ${ }^{3}$, Hong G. Im $^{3}$ \\ ${ }^{1}$ Department of Aerospace Engineering, Indian Institute of Science, \\ Bangalore-560012, India. \\ ${ }^{2}$ National Center for Combustion Research and Development, Indian Institute of Science, \\ Bangalore-560012, India. \\ ${ }^{3}$ Clean Combustion Research Center, King Abdullah University of Science and Technology, \\ Thuwal-23955, Saudi Arabia.
}

\begin{abstract}
Interactions of turbulence, molecular and energy transport coupled with chemistry play a crucial role in the evolution of flame surface geometry, propagation, annihilation and local extinction/re-ignition characteristics of intensely turbulent premixed flames. This study seeks to understand how these interactions affect flame surface annihilation of lean hydrogen-air premixed turbulent flames. Direct numerical simulations (DNS) are conducted with detailed reaction mechanism and transport properties for hydrogen-air flames, at different parametric conditions. Flame particle tracking (FPT) technique is used to follow specific flame surface segments. An analytical expression for the local displacement flame speed $\left(S_{\mathrm{d}}\right)$ of a temperature isosurface is considered and the contributions of transport, chemistry and kinematics on the displacement flame speed at different turbulence-flame interaction conditions are identified. In general, the displacement flame speed for the flame particles is found to increase with time for all conditions considered. This is because, eventually all flame surfaces and their resident flame particles approach annihilation by reactant island formation at the end of stretching and folding processes induced by turbulence. Principal curvature evolution statistics obtained using FPT suggest that these islands are ellipsoidal on average, enclosing fresh reactants. Further examinations show that the increase in $S_{\mathrm{d}}$ is caused by the increased negative curvature of the flame surface and eventual homogenization of temperature gradients, as these reactant islands shrink due to flame propagation and turbulent mixing. Finally, the evolution of the normalized, averaged, displacement flame speed vs. stretch Karlovitz number was found to collapse on a narrow band, suggesting that a unified description of flame speed dependence on stretch rate may be possible in the Lagrangian description.
\end{abstract}

Keywords: Direct numerical simulation; turbulent premixed flames; displacement flame speed; flame particle tracking

"E-mail addresses: schaudhuri@aero.iisc.ernet.in, swetaprovo@gmail.com 


\section{Introduction}

Turbulent premixed combustion is commonly described as an ensemble of laminar flames segments, which are locally stretched and wrinkled by the interaction of turbulent mixing and chemical reactions. While the local flame structure consists of transport and reaction zones with finite thicknesses, the front propagation is often represented by the dynamics of isoscalar surfaces based on temperature or mass fraction of a chosen species [1]. This approach allows a systematic way to identify the distributions of local flame propagation speed, along with various surface kinematic quantities. Such a framework has been widely employed in two-dimensional direct numerical simulation (DNS) studies considering both detailed chemistry for hydrogen and hydrocarbon fuels [2-4], in which the effects of strain rate and curvature on the local displacement speed were extensively investigated, and estimates of the effective Markstein number according to the laminar flame theory were attempted. Earlier three-dimensional DNS studies using a single step chemistry model systematically explored the effects of Lewis [5] and Damköhler numbers [6]. The local flame geometries were also investigated, and the effects of strain rate and curvature on different regions across the flame were analyzed in detail [7, 8]. Large scale DNS at higher Reynolds and Karlovitz numbers considering detailed chemistry have also been conducted [9-13], with an emphasis on the fractal characteristics.

The above studies mainly investigated the local flame topology and kinematics with the analysis applied to an instantaneous solution field. While these studies have provided valuable insights into the fundamental understanding of the global statistical characteristics of turbulent flames, such an Eulerian one-time analysis lacks in tracking temporal history of specific events. As such, the Eulerian analysis is not adequate in describing highly localized phenomena such as strain/curvature induced extinction, mutual annihilation of flame surfaces and pocket formation [14], which are believed to be critical aspects in predicting the flame stabilization and pollutant formation. For this purpose, a Lagrangian approach monitoring the evolution of a specific surface element during the critical events could be more appropriate.

In non-reacting turbulent flows, the Lagrangian description has been adopted as a rational way to understand the effects of turbulent transport characteristics [15-17], since the turbulent mixing and dispersion in the inertial range arises from advection of the velocity fluctuations [17]. A general mathematical description of the evolution of surfaces in turbulent flows, developed by Pope [18] established rigorous formulation to describe the Lagrangian evolution of surface elements in turbulent flows, in a form directly applicable to propagating 
surfaces. Following this approach, the present study employs a flame particle tracking (FPT) technique, introduced in [19], which allows a systematic investigation of the temporal evolution of multiple locations on isoscalar surfaces. This is achieved by tracking flame particles represented by a set of discrete points which are instantaneously moving in the direction normal to the surface at the local displacement speed $[2,3]$ relative to the local fluid velocity. As such, the evolution of the position vector of a flame particle residing on these isoscalar surfaces at any instant of time is described by,

$\frac{d}{d t} \mathbf{X}^{F}(t)=\mathbf{U}\left(\mathbf{X}^{F}[t], t\right)+S_{\mathrm{d}}\left(\mathbf{X}^{F}[t], t\right) \mathbf{n}^{F}\left(\mathbf{X}^{F}[t], t\right)$

where $\mathbf{X}^{F}(t)$ is the flame particle position at time $t, \mathbf{U}\left(\mathbf{X}^{F}[t], t\right)$ is the local flow velocity at $\mathbf{X}^{F}(t), S_{\mathrm{d}}\left(\mathbf{X}^{F}[t], t\right)$ is the local displacement speed of the isosurface, and $\mathbf{n}^{F}\left(\mathbf{X}^{F}[t], t\right)$ is the local unit vector normal to the isosurface.

As will be elaborated later, the displacement speed, $S_{\mathrm{d}}$, defined based on a given scalar variable $[2,3]$, is the most important quantity that determines the local propagation of the flame front and the flame structure in general. Therefore, the Lagrangian analysis on the contributions of the individual terms to $S_{\mathrm{d}}$ allows an identification of the key mechanisms for the turbulence-flame interaction. The time history and statistics of these flame particles provide important physical insights regarding the flame surface annihilation and local extinction mechanism. The technique is also used to study the effects of evolving kinematic properties, such as tangential, normal strain rates and principal curvatures during annihilation.

In the present work, the FPT technique developed in Ref. [19] is applied to DNS of statistically planar premixed flames interacting with the approaching near isotropic turbulence. This study analyzes the combined effects of turbulence, transport, chemistry and kinematic properties of premixed flames, which can be significantly altered by the flame topology during flame surface annihilation and extinction. Turbulence leads to flame surface stretching and folding, thereby increasing the flame surface area. However, inherent flame responses such as kinematic restoration, Markstein diffusion and pocket formation lead to flame surface destruction. It is also noted that the chemical reaction and heat release support the scalar gradient while the diffusive nature of turbulence tries to dissipate the gradients. These competing phenomena ensure that the turbulent flame speed and surface areas do not exhibit unbounded growth and maintain near statistically stationary behavior. Under these 
conditions, the Lagrangian analysis seeks a unified description of $S_{\mathrm{d}}$ by examining the evolving negative curvature regions of the temperature isosurfaces.

\section{Computational Approach}

\section{a. Direct Numerical Simulations}

Three dimensional (3D), reacting flow DNS are performed in an inflow-outflow configuration, as shown in Figure 1a. The computational domain is a rectangular box. The Navier-Stokes characteristic boundary conditions (NSCBC) [20-22] were used in inflow and outflow direction ( $x$-direction), while $y$ and $z$ directions were maintained periodic. Figure $\underline{1 b}$ shows an instantaneous flame surface image from a representative simulation for Case 3 , where the heat release rate is extracted on the temperature isosurface.

The simulations were carried out at four different levels of turbulence intensities, which resulted in different $R e_{T}, D a$ and $K a$ conditions. Cases 1-4 have been named in the order of increasing $\mathrm{Ka}$. The details of computational and physical parameters are listed in Table $\underline{1}$. Figure 2 shows the parametric conditions for the four cases on a regime diagram, indicating that Case 1 lies in the corrugated flamelet region, while Cases 2-4 represent the thin reaction zones regime.

Simulations for Cases 1, 3 and 4 were performed using the Pencil code [23], an open source code which solves the continuity, momentum, energy (temperature form) and species transport equations in fully compressible formulation on a structured 3D Cartesian geometry, with a sixth-order explicit finite difference scheme for spatial discretization, and a third-order Runge-Kutta scheme for time integration. The transport properties are evaluated using the mixture averaged formulation. The detailed description of evaluations of these properties and other relevant parameters are provided in Ref. [24]. A detailed $\mathrm{H}_{2}$-air chemical reaction mechanism consisting of 9 species and 19 reactions [25] was implemented and validated in the Pencil code.

As an additional simulation dataset, Case 2 was produced using the $\mathrm{S} 3 \mathrm{D}$, whose detailed numerical algorithms can be found in previous studies [26]. The configuration and boundary conditions are consistent as in Figure 1a. Other physical parameter conditions are slightly different, as shown in Table $\underline{1}$. For Case 2 , the detailed $\mathrm{H}_{2}$-air chemical reaction mechanism developed by Burke et al. [27] consisting of 9 species and 19 reactions was used.

\section{b. Flame Particle Tracking Algorithm}


The DNS results obtained from Cases 1-4 serve as an input to the FPT algorithm. In the FPT technique, a reactive isoscalar surface is chosen and the distinct locations on the surface are marked. These marked mathematical points are referred to as the flame particles, which always remain embedded on, and move with the corresponding isoscalar surface. The time evolution of a flame particle location is governed by Eq. (1), which is integrated over time using the triangle-ray intersection algorithm [28]. Since the flame particles are passive, the integration is performed by post-processing the stored DNS data fields. A Runge-Kutta type predictor-corrector scheme is used to determine the flame particle velocity. Further details of the FPT algorithm can be found in Ref. [19].

In the present paper, a planar, temperature isosurface is chosen as the initial condition for the Case 1 FPT simulation, whereas for Case 3, a moderately wrinkled temperature isosurface is considered. Curvature and tangential strain rate evolutions for Case 1 and 3 could be found in [19]. As such, the FPT results for Cases 1 and 3 reveal the developing phase of the flame surface behavior. On the other hand, for Case 4 reported in [29] and newly computed Case 2, a highly wrinkled temperature isosurface with detached flame islands is chosen as the initial condition for the FPT simulation, such that the turbulent flame has already reached a statistically stationary condition. The intention was to explore both developing and mature state of turbulence-flame interaction characteristics given a finite particle lifetime.

Various parameters, such as divergence of heat flux, heat release rate, reaction rate, curvature, and strain rate, are calculated for each flame particle resident on a given isoscalar surface. These parameters are interpolated from the DNS grid points onto the isosurface mesh using the Matlab ${ }^{\circledR}$ built-in marching cubes algorithm. Subsequently, these parameters are interpolated from surface mesh to the flame particle locations.

\section{c. Choice of Isoscalar Surface and Iso-value}

The kinetic effects based on the FPT analysis were examined in terms of the two most important reactions of $\mathrm{H}_{2}$-air chemistry:

$$
\begin{aligned}
& \mathrm{H}+\mathrm{O}_{2}=\mathrm{OH}+\mathrm{O} \\
& \mathrm{H}+\mathrm{O}_{2}+\mathrm{M}=\mathrm{HO}_{2}+\mathrm{M}
\end{aligned}
$$

The reactions $\mathrm{R} 1$ and $\mathrm{R} 9$ are well-known chain branching and third body reactions, respectively [30]. R1 has a large activation energy and is highly sensitive to temperature, while R9 has almost zero activation energy. As such, the laminar flame speed and ignition 
delay time are highly sensitive to reaction $\mathrm{R} 1$, which serves a sink for the $\mathrm{O}_{2}$ molecules and a source for more radicals. The radical species produced by breaking the fuel molecules help in the overall progress of the reaction. The three body recombination reaction $\mathrm{R} 9$, characterized by zero activation energy is the major contributor to the exothermicity of the overall reaction mechanism. Recognizing the importance of reactions R1 and R9, in the present work, flame particles are chosen to be resident on temperature isosurfaces. The expression for the reaction rate for the $j^{\text {th }}$ reaction is given as [30]:

$\dot{\omega}_{j}^{\prime \prime \prime}=k_{j, f} \prod_{\alpha=1}^{N} C_{\alpha}^{v_{\alpha, j}^{\prime}}-k_{j, r} \prod_{\alpha=1}^{N} C_{\alpha}^{v_{\alpha, j}^{\prime \prime}}$

where, $k_{j, f}$ is specific reaction constant of a forward reaction and is given by:

$k_{j, f}=A_{j} T^{\beta_{j}} e^{-\left(\frac{E_{a, j}}{R_{u} T}\right)}$

For a temperature isosurface, evaluation of Eq. (3) becomes straightforward as the exponential term is constant. This also simplifies any modeling of specific reaction rates instantaneously as well as in the statistical average. Fig. $\underline{3}$ plots the reaction rates of R1 and R9 as a function of temperature for $\phi=0.81$ obtained from the PREMIX code [31]. It is seen that the reaction rates of $\mathrm{R} 1$ and $\mathrm{R} 9$ peaks at $T=1321 \mathrm{~K}$ and $T=665 \mathrm{~K}$, respectively. Thus, these values of temperatures are chosen for this study. The behavior is nearly identical for Case 2 at a slightly leaner condition $(\phi=0.7)$, and the same temperature isovalues were used for the subsequent analysis.

In the present study, the displacement flame speed, $S_{d}$, is defined as the speed of a given isosurface in the direction normal to itself relative to the local flow velocity. As such, $S_{d}$ varies depending on the choice of the temperature iso-values. For temperature isosurface the equation for $S_{\mathrm{d}}$ is given by,

$$
S_{d}=\frac{1}{\rho c_{p}|\boldsymbol{\nabla} T|}[\underbrace{\boldsymbol{\nabla} \cdot(\lambda \boldsymbol{\nabla} T)}_{\text {Term } 1}+\underbrace{\rho \boldsymbol{\nabla} T \cdot \sum_{\alpha}\left(D_{\alpha} c_{\mathrm{p}, \alpha} \boldsymbol{\nabla} Y_{\alpha}\right)}_{\text {Term } 2}-\underbrace{\sum_{\alpha} h_{\alpha} \dot{\omega}_{\alpha}^{\prime \prime \prime}}_{\text {Term } 3}]
$$

where the local unit normal vector $(\mathbf{n})$ is defined as positive towards the unburned mixture as:

$\mathbf{n}=-\frac{\boldsymbol{\nabla} T}{|\boldsymbol{\nabla} T|}$ 
Equation (4) is derived by neglecting pressure and frictional work term, which is valid at low Mach number conditions as in the present study.

To validate that the FPT algorithm is predicting the correct displacement speed, the initial condition for Case 1, at which the temperature isosurfaces corresponding to the two temperature iso-contours are nearly planar, are considered. The $S_{\mathrm{d}}$ for each flame particle residing on these isosurface is then calculated using the Eq. (4). The ensemble average of $S_{\mathrm{d}}$ at this instant is then compared with the planar laminar flame speed at a given temperature location, denoted as $S_{L, T}$, obtained from the CHEMKIN PREMIX calculations. The comparison is shown in Table 2 . For both temperature iso-values, at $665 \mathrm{~K}$ and $1321 \mathrm{~K}$, the calculated $S_{\mathrm{d}}$ from FPT is in close agreement with the $S_{L, T}$, with an observed discrepancy of $2 \%$ and $4 \%$, respectively. Therefore, the accuracy of FPT algorithm is validated.

\section{Results and Discussion}

First, the conventional results of flame surface statistics are presented in terms of curvature and strain rate obtained over the entire temperature isosurface. Figure $\underline{4}$ plots the probability density function (pdf) of mean curvature $(\kappa)$, tangential strain rate $\left(K_{\mathrm{t}}\right)$, and normal strain rate $\left(K_{\mathrm{N}}\right)$, at the last time step of FPT analysis $\left(t=\tau_{\text {track }}\right)$ for the entire temperature isosurface of (a) $665 \mathrm{~K}$ and (b) $1321 \mathrm{~K}$. Four simulation cases with different choices of the initial fields are compared. Flame surface convex (concave) towards the reactant side is defined as having a positive (negative) curvature. For all cases, for both temperature isovalues, the curvature pdfs are symmetric about the mean at nearly zero, consistent with previous studies [3, 4]. The pdfs of $K_{\mathrm{t}}$ and $K_{\mathrm{N}}$ show shift in the peak towards the positive side, again consistent with Refs. [5, 6] and other related studies. This is attributed to the preferential alignment of the principal strain rates and the local scalar gradients [32, 33].

The JPDFs of $\kappa_{1}$ and $\kappa_{2}$ obtained according to the condition $\kappa_{1}<\kappa_{2}$, at $t=\tau_{\text {track }}$, for the entire $665 \mathrm{~K}$ and $1321 \mathrm{~K}$ iso-temperature surfaces are shown in Figs. $\underline{5}$ and $\underline{6}$, respectively. It is found that for both the iso-temperature surfaces the principal curvatures $\kappa_{1}$ and $\kappa_{2}$ are mostly positively correlated, with shapes consistent with those found by Shim et al. [13]. The JPDF of $\kappa_{1}$ and $\kappa_{2}$ obtained by sorting according to $\left|\kappa_{1}\right|<\left|\kappa_{2}\right|$ for $1321 \mathrm{~K}$ temperature isosurface could be found in appendix $\underline{A}$.

The above results provide the distribution of the kinematic properties over the entire temperature isosurface, not only at the flame particle locations. To understand the geometry 
of the flame islands, it is important to study the time evolution of principal curvatures at the flame particle locations, which will be explained in the following sub-section.

In Ref. [19], it was found that for Cases 1 and 3, both the tangential strain rate and the mean curvature increase to large positive and negative values, respectively, as the flame surface elements were annihilated. These observations are attributed to the reorientation of local surface tangents towards the most extensive component of the principal strain rate. Consequently, the tangential strain rate of the flame particle ensemble continues to increase and results in formation of negative curvature regions. Simultaneously, this also facilitates adjacent surface collision leading to reactant island formation surrounded by product gases. These detached flame islands behave similar to inwardly propagating spherical flame. In these islands, the trapped reactants are consumed and the flame surface propagates normal to itself consequently resulting in flame surface and flame particle loss. This process is however further complicated by the background turbulent mixing. It was also shown that, towards the end of flame particle ensemble lifetime, the local displacement flame speed $\left(S_{\mathrm{d}}\right)$ increases sharply. In a recent computational work with single step chemistry, Lipatnikov et al. [34] observed such an increased flame speed in the "unburnt mixture finger" regions. Similar observations were reported in Chen et al. [14] using 2D DNS of lean methane-air flames.

In the present study, the transport and chemistry effects are delineated by investigating the different terms that constitute $S_{\mathrm{d}}$ in Eq. (4). This will allow us to determine which among these terms play a dominant role in the flame surface annihilation in different regimes of interest and hence assist in future modeling efforts. FPT technique offers us the feasibility to investigate the time evolution of each of the individual effects quantitatively for surface locations uniquely identified in time. By definition of FPT, these observations are conditional upon the choice of the scalar and the isoscalar value representing the isoscalar surface. The following results are obtained by tracking one thousand flame particles, which were initially uniformly distributed on two thirds of the surface area of temperature isosurface. This ensured that flame particles did not move out of the $y-z$ boundaries, over the tracking time.

Figures $\underline{7}$ and $\underline{8}$ show the probability density function (pdf) of the lifetime $\left(\tau_{F, L}\right)$ of flame particles residing on $665 \mathrm{~K}$ and $1321 \mathrm{~K}$ temperature isosurfaces, respectively, for Cases 1-4. For Cases 1, 3 and 4, the majority of the flame particles survive through to the end of the tracking time. For Case 2, however, few particles survive until the end of the tracking time. This is because higher turbulence intensity increases the wrinkling of a flame surface and 
thereby increases the loss of flame particles by flame surface annihilation. Based on the these observations, for Cases 1, 3 and 4, flame particles with lifetime in the range $0.5 \leq \tau_{F, L} / \tau_{\text {track }}<1.0$ are chosen, while for Case 2, a slightly larger range of lifetime $0.25 \leq \tau_{F, L} / \tau_{\text {track }}<1.0$ is preferred in order to obtain meaningful statistics. Results from the analysis of the two different temperature isosurfaces are summarized in the following.

\section{a. Analysis of the $T=665 \mathrm{~K}$ isosurfaces}

Here, we analyze the different properties of flame particles as a function of a scaled time. In Figs. $\underline{9-22}$ (except Figs. $\underline{14}$ and 16), the red lines represent the variation in the property of individual flame particle and the solid black lines represent the mean value of the quantity averaged over all the flame particles at a particular time instant $\left(t / \tau_{F, L}\right)$. The dotted black lines represent the standard deviation of a variable about the mean. Here, the normalized time $\left(t / \tau_{F, L}\right)$ is defined as the ratio of the time $(t)$ to lifetime of the corresponding flame particle $\left(\tau_{F, L}\right)$ [19]. A zero value of normalized time corresponds to the time instant at which the flame particle is inserted onto the isosurface, while a unity value represents the time instant at which the flame particle is lost from the isosurface.

The time evolution of $S_{\mathrm{d}}$ is shown in Fig. $\underline{9}$. It is observed that the individual flame particles and their ensemble average (solid black lines) show a rapid increase in the $S_{\mathrm{d}}$ towards the end of their lifetime. These results are consistent with recent studies [14, 19, 34]. The sharp increase in $S_{\mathrm{d}}$ can be reasoned to either diffusion or curvature effects, which are examined as below.

To assess the above behavior of $S_{\mathrm{d}}$, the individual terms in Eq. (4) are examined in detail. Terms 1 through 3 represent the contributions from the heat conduction, heat transport via species diffusion, and the heat release rate, respectively. All these terms are affected by molecular as well as turbulent transport.

Figure 10 show the time evolution of Term 1, the heat flux contribution, for Cases 14. For all cases, Term 1 increases for both individual flame particles and the statistical average as the flame particles reach the end of their lifetime. The fluctuations also increase with increasing $\mathrm{Ka}$, implying an increased relative effect of turbulence on wrinkling of temperature isosurfaces. The flame particles, initially distributed uniformly on the isosurface, become concentrated in the negative curvature regions of the temperature isosurface over time. These regions are subjected to the focusing of thermal energy. The reactant mixture ahead of the temperature isosurface is heated up and the gradients reduce. This leads to a 
decrease in the heat flux leaving the control volume bounding the temperature isosurface. Hence the divergence of heat flux increases as the flame particles approach the end of their lifetime. Similar observations are made in the recent work of Fogla et al [35] and Poludnenko and Oran [36] using 2D and 3D DNS of premixed flames respectively, using Eulerian analysis.

Figure 11 show the evolution of Term 2, the mass diffusion contribution to thermal transport, as a function of the normalized time for Cases 1-4. By comparing the absolute magnitude among the three terms, it is evident that the effect of Term 2 is negligible for all cases, consistent with Altantzis et al. [37].

The effect of Term 3, the contribution of the heat release rate on the behavior of $S_{\mathrm{d}}$, is presented in Fig. 12. It is found that the mean heat release rate increases weakly for Cases 1 and 3, remains nearly constant for Case 4 with normalized time. For Case 2, Term 3 remains almost constant, with a small decrease at the end of normalized time. These observations suggest that the effect of heat release rate on the increase of $S_{\mathrm{d}}$ is insignificant compared to that of heat conduction.

The last variable that influences the $S_{\mathrm{d}}$ is magnitude of temperature gradient $(|\nabla T|)$, which may be affected by: (i) a change in local temperature due to heat release, (ii) bringing two temperature isosurfaces closer to each other, and (iii) by changing the local curvature. (i) can be considered as a kinetic effect, while (ii) and (iii) could be termed as kinematic effects. Time evolutions of $|\nabla T|$ are shown in Fig. 13. For all cases, $|\nabla T|$ for the individual flame particle and their ensemble mean decreases sharply towards the end of lifetime. From the above analysis it is clear that both Term 1 and $|\nabla T|$ are the dominant parameters contributing towards increasing $S_{\mathrm{d}}$. The significance of Term 1 is analyzed above. Furthermore, the influence of $|\nabla T|$ on $S_{\mathrm{d}}$ is investigated as follows.

The joint evolution of $S_{\mathrm{d}}$ and $|\nabla T|$ with the normalized time is plotted in Fig. 14. A negative correlation is observed between $S_{\mathrm{d}}$ and $|\nabla T|$ with the increasing $t / \tau_{F, L}$. Chen et al. [38] reported a similar correlation for the conditions pertaining to that of simplified HCCI engine configuration. Considering that the heat release rate remains almost constant throughout the lifetime of the flame particles, the contribution of heat release rate to reduce $|\nabla T|$ for flame particles of the $T=665 \mathrm{~K}$ isosurface is negligible.

The second factor that influences $|\nabla T|$ is the normal strain rate $\left(K_{\mathrm{N}}\right)$, as shown in Fig. 15. The extensive normal strain rate $\left(K_{\mathrm{N}}>0\right)$ increases the distance between the two adjacent temperature isosurfaces and thereby decreases $|\nabla T|[8]$, while $K_{\mathrm{N}}<0$ increases $|\nabla T|$ by 
drawing the temperature isosurfaces closer. For the $665 \mathrm{~K}$ isosurface, the plots of $K_{\mathrm{N}}$ versus normalized time show a rather weak variation for all four cases. This indicates that the effect of $K_{\mathrm{N}}$ in diminishing $|\nabla T|$ is also negligible.

This leaves the local curvature effect to be the main mechanism to affect $|\nabla T|$. To illustrate this effect, the joint evolution of $|\nabla T|$ vs. $\kappa$ with normalized time is plotted in Fig. 16. The quantities $|\nabla T|$ and $\kappa$ exhibit a correlation showing an increase in curvature magnitude in the negative direction and simultaneous decrease of $|\nabla T|$ with the normalized time. For all cases, magnitude of the mean curvature for the individual flame particle and ensemble mean increases in the negative direction towards the end of the normalized time. This confirms the reasoning that the curvature effect is dominant in the variation in $|\nabla T|$.

For a stationary spherical flame with the reactants entering from a point source which is surrounded by the flame, the non-dimensional unburnt mass flux and non-dimensional curvature are related as [30]:

$\tilde{f}_{u}=\left(1+\frac{1}{2} \widetilde{\nabla} \cdot \mathbf{n}\right)^{-2}$

$\tilde{f}_{u} \approx(1-\widetilde{\nabla} \cdot \mathbf{n})$

$\tilde{\kappa}=\widetilde{\nabla} \cdot \mathbf{n}=\widetilde{\nabla} \cdot\left(-\frac{\widetilde{\nabla} T}{|\widetilde{\nabla} T|}\right)$

where $\widetilde{\nabla}=\delta_{L} \nabla$ and $\tilde{\kappa}=\delta_{L} \kappa$, with $\delta_{L}$ being the flame thickness.

Eq. (6) is the non-dimensional mass flux and Eq. (8) is the non-dimensional curvature. Eq. (7) is the linearized form of Eq. (6) [30], indicating that the flame speed increases for the negatively curved flames as observed in the present study. For the conditions considered in the present work, magnitude of the mean curvature increases towards the negative direction as the flame particles reach the annihilation state (Fig. 16). The increase in curvature enhances the relative upstream mass flux through Eq. (7) and thereby accelerates $S_{\mathrm{d}}$ to very large values as the flame particles reach the end of their lifetime. These observations suggest that the curvature without stretch can also solely affect the local flame speed.

To assess the geometry of the isolated islands formed towards the end of the flame particle lifetime, it is important to study the evolution of $\kappa_{1}$ and $\kappa_{2}$. Using the 2D DNS data, Chen et al. [14] implicitly studied isolated islands that are of cylindrical geometry. Shim et al. [13] also classified the flame shapes based on the JPDF of $\kappa_{1}$ and $\kappa_{2}$. These two important studies, however, did not consider time evolution of the $\kappa_{1}$ and $\kappa_{2}$ at distinct locations on the 
isoscalar surface. Fig. 17 plot the time evolution of the principal curvatures $\left(\kappa_{1}\right.$ and $\kappa_{2},\left|\kappa_{1}\right|<\left|\kappa_{2}\right|$ ) for Cases 1-4, showing that both quantities drops to negative values toward the end of lifetime which suggests that the geometry of the isolated reactant islands formed near the end of flame particle lifetime is ellipsoidal on statistical average.

\section{b. Analysis of the $T=1321 \mathrm{~K}$ isosurfaces}

In this sub-section, the results obtained by tracking the flame particles residing on $1321 \mathrm{~K}$ temperature isosurface are discussed. As shown in Fig. $\underline{3}, T=1321 \mathrm{~K}$ represents the surface corresponding to the maximum rate of the branching reaction $\mathrm{R} 1$. The analysis procedure here follows that of $T=665 \mathrm{~K}$. However, consideration of another temperature isosurface adds to the generality of observations, or lack thereof, depending on the observables of interest.

The evolution of $S_{\mathrm{d}}$ with normalized time for $T=1321 \mathrm{~K}$ are plotted in Fig. $\underline{18}$. The overall trend is similar to the results in Fig. 9 , in that $S_{\mathrm{d}}$ increases rapidly towards the end of the lifetime. While the statistical mean behavior appears similar, the individual flame particle behavior in Fig. 18 shows a greater level of fluctuations, more pronounced at higher Karlovitz number. Figures $\underline{19}-\underline{21}$ show the individual contributions of Terms 1 through 3. Most of these results are consistent with those shown in Figs. 10-12, with a few notable differences. First, unlike in Fig. 10, Fig. 19 show that Term 1 starts with a negative value and increases to large positive value towards the end of the flame particle lifetime. The sharp increase of heat flux towards end of flame particle lifetime is attributed to thermal focusing, as explained in the previous subsection. Fig. 20 shows that the heat transport due to species diffusion is insignificant, consistent with the result in Fig. 11 . However, the results in Fig. 21 demands special attention, in that heat release rate decreases in time for all four cases. Unlike the $665 \mathrm{~K}$ isosurface which quickly vanishes, the $1321 \mathrm{~K}$ isosurface survives relatively longer to exhibit heat release attenuation through the flame annihilation process due to island formation. These flame islands are filled with a small amount of unburned mixture and are embedded in high temperature products. In these islands, as the reactions progress the flame propagates inwardly until the fresh reactants enclosed by the isosurface are completely consumed. Thus, eventually depletion of reactants causes the heat release rate to decrease towards the annihilation of these flame islands.

The effects of $|\nabla T|$ shown in Fig. 22 also reveal consistent behavior as in Fig. 13, and the same analysis was undertaken in order to examine different factors contributing to $|\nabla T|$. 
The results are consistent with the $T=665 \mathrm{~K}$ case, in that the effect of $K_{\mathrm{N}}$ is insignificant, and the magnitude of curvature shows a sharp increase in the negative direction near the end of the flame particles' lifetime along with a negative correlation with $|\nabla T|$. The main difference is found in the larger effect of the heat release rate on $|\nabla T|$ for the $T=1321 \mathrm{~K}$, as was shown in Fig. 21. This is further confirmed by Fig. 23, showing a much stronger correlation between $|\nabla T|$ and $S_{\mathrm{d}}$ with a lower level of scatter. This implies that for the $T=1321 \mathrm{~K}$, the effect of turbulent mixing leading to dissipation of gradients is more directly accompanied by a reduced heat release rate, resulting in a stronger impact on the displacement flame speed. In contrast, at the $T=665 \mathrm{~K}$ isosurfaces, heat release rate always remain much stronger and $S_{\mathrm{d}}$ is increased through curvature and associated kinematics.

The effect of turbulence-induced curvature on the attenuation of the heat release rate is illustrated in Fig. 24, showing the snapshots of the temperature distribution for Cases 1-4, respectively. The black box in these figures qualitatively tracks the disappearance of flame islands by propagation and mixing. It is seen that the high temperature contours of the reactant islands survive longer, leaving temperature in-homogeneities in the otherwise product field. Hence, reactant depletion and heat release rate reduction is more pronounced near the end of lifetime for the $T=1321 \mathrm{~K}$ isosurface flame particles in comparison to those for the $T=665 \mathrm{~K}$ isosurfaces.

Finally, geometry of the isolated islands of the $T=1321 \mathrm{~K}$ isosurfaces was investigated. Without showing the results, it is reported that the geometry of reactant filled islands are statistically ellipsoidal for all cases, consistent with the results for the $T=665 \mathrm{~K}$ isosurfaces.

\section{Effect of the stretch Karlovitz number}

In this section, characterization of effect of turbulence intensity on $S_{d}$ is attempted by analyzing the local Karlovitz number determined at the flame particles by employing the FPT technique. To distinguish from the turbulent Karlovitz number $(\mathrm{Ka})$ used in the regime identification, the stretch Karlovitz number $\left(K a_{S}\right)$ is defined on an isosurface as [3]:

$K a_{s}=\frac{\delta_{L}}{S_{L, T}}\left(K_{t}+S_{d} \boldsymbol{\nabla} . \boldsymbol{n}\right)=K a_{T}+K a_{C}$

where $K_{t}$ is the tangential strain rate. The two terms on the RHS of Eq. (9) are referred to as the tangential and curvature Karlovitz number, respectively. In [3] $S_{\mathrm{d}}$ was evaluated on a species isosurface and the study was carried out at different turbulence intensity levels. 
The behavior of $\left\langle S_{d} / S_{L, T}\right\rangle$ with $\left\langle K a_{T}\right\rangle,\left\langle K a_{C}\right\rangle$ and $\left\langle K a_{S}\right\rangle$ are shown in Figs. $\underline{25}$ and $\underline{26}$ for the $665 \mathrm{~K}$ and $1321 \mathrm{~K}$ isosurfaces, respectively. Here the brackets represent averaging over the entire flame particle ensemble throughout their lifetime. For both temperature isosurfaces, the following two observations are noted: first, the effect of curvature is dominant over tangential strain in dictating the overall stretch Karlovitz number effect. Second, the $\left\langle S_{d} / S_{L, T}\right\rangle$ vs. $\left\langle K a_{S}\right\rangle$ for all cases collapse to a strong correlation curve, irrespective of the initial condition and turbulence intensity levels considered. Furthermore, the correlation appears to be slightly nonlinear, but the deviation from linearity is only observed at large negative curvature conditions, which are limited to a smaller fraction of the total flame particles. This suggests that, from a Lagrangian description of the flame front, a linear relationship between $S_{\mathrm{d}}$ and $K a_{S}$ may still serve as a good approximation in describing turbulent premixed flame dynamics.

\section{Implications in turbulent combustion modeling}

The previous section highlights the marked difference in the flame speed versus stretch correlations reported from the traditional Eulerian analysis showing large scatters e.g. [3]. These models were based on an one-time snapshot of the data fields using a quasi-steady assumption $[35,39]$. Due to the difficulties in fitting the data into a linear correlation, subsequent studies developed models to separate different Markstein numbers for the tangential strain and curvature components as suggested from laminar flame studies [40-42]. Even with such a modified approach, the validity of such models are often limited to weak stretch conditions. The present FPT analysis demonstrated much stronger correlations based on Lagrangian description of the flame particles, suggesting that the larger scatter in the Eulerian analysis may be attributed to the history or non-local effects that cannot be captured by the analysis of one-time data field. By systematically accounting for the temporal evolution of individual flame segments, the FPT approach yielded much stronger correlations. While the FPT analysis yielded a mild level of nonlinearity in the correlations, Figs. 25 and 26 suggested that the correlations are valid for different parametric conditions as well as different choices of isosurfaces, thereby extending the accuracy and generality of the model.

To utilize the present Lagrangian approach to build a framework for closure of turbulent combustion models based on the flamelet concept, consider the transport equation for the flame surface density function as written in Ref. [43]: 
$\frac{\partial \Sigma^{\prime}}{\partial t}+\nabla \cdot \dot{X} \Sigma^{\prime}=\dot{S} \Sigma^{\prime}$

where $\dot{X}=V_{f}=\boldsymbol{U}+S_{d} \boldsymbol{n}$ and $\dot{S}=K_{t}+S_{d} \nabla . \boldsymbol{n}$.

Here, $S_{d}, \boldsymbol{U}, \dot{S}$, and $K_{t}$ are the flame propagation speed, fluid velocity, flame stretch rate and flow tangential strain rate, respectively. To make the computation of the flame surface density variable explicitly dependent on turbulence and chemistry, the mean curvature must also be computed from these quantities. Without computing quantities for the isosurface and its associated properties at each time step, this can be achieved by an on-the-fly FPT framework using the deterministic evolution equation for the principal curvatures. For example, the evolution equation for the principal curvature, $K_{1}$, is written as [18]:

$\dot{K}_{1}=\left\{\frac{\partial^{2}\left(S_{d}+U_{3}\right)}{\partial y_{1}^{*} \partial y_{1}^{*}}\right\}^{0}+\left(K_{N}-2 S_{11}^{*}\right) K_{1}+S_{d}^{o} K_{1}^{2}$

where the first term on the RHS is evaluated at a point on a surface. The evolution of the principal curvatures in turn depends on the evolution of the local coordinate on which these evolution equations are defined. In stochastic models [44], the local expected burning rate is a product of local propagation speed, $S_{d}$ and the expected flame surface density. Thus, the modelling efforts for $S_{d}$ in the Lagrangian viewpoint presented in this article can strongly contribute towards the computation of these important quantities. The improved computation of the above quantities can further assist in developing more robust and accurate sub-grid scale models for large eddy simulation (LES) and stochastic models for premixed turbulent combustion.

\section{Conclusions}

DNS of lean $\mathrm{H}_{2}$-air premixed combustion is performed in an inflow-outflow channel type configuration in order to study the turbulence-transport-chemistry interaction for statistically planar premixed flames interacting with near isotropic turbulence. Detailed chemistry involving 9 species and 19 reactions is used. FPT along with DNS is used to study the propagation characteristics of two temperature isosurfaces.

The present study aims to understand $S_{\mathrm{d}}$ in evolving negative curvature regions of temperature isosurfaces within turbulent premixed flames. Time evolution of $S_{\mathrm{d}}$ is studied by considering two different temperature isosurfaces $(665 \mathrm{~K}$ and $1321 \mathrm{~K}$ corresponding to peak 
reaction rates of reactions $\mathrm{R} 9$ and $\mathrm{R} 1$ respectively) each interacting with four distinct levels of turbulence intensity. Individual terms of the local displacement flame speed as given by analytical expression Eq. (4) are evaluated to assess their bearing on the behavior of $S_{\mathrm{d}}$. The effects of turbulent mixing and curvature on the propagation characteristics of the flame front are highlighted. The analysis of the individual terms of Eq. (4) leads to the conclusion that the rapid increase in $S_{\mathrm{d}}$ towards the end of the flame particle lifetime is attributed to two phenomena: (a) the increase in the divergence of heat flux (Term 1) and (b) the decrease in $|\nabla T|$.

The increase in the divergence of heat flux towards the end of flame particles' lifetime is attributed to the concentrating heat flow in the negative curvature regions of the temperature isosurface. The effects of heat transfer by species diffusion was found to be insignificant in contributing to the increased displacement flame speed for all cases considered. The $S_{\mathrm{d}}$ evolution on $665 \mathrm{~K}$ isosurface is weakly sensitive to heat release rate, whereas that on the $1321 \mathrm{~K}$ isosurface is largely affected by reduced heat release rate. This decrease in the heat release rate in normalized time is due to the depletion of the reactants in the isolated islands surrounded by higher temperature product gases.

The joint evolution of $S_{\mathrm{d}}$ and $|\nabla T|$, with normalized time also shows a negative correlation between $S_{\mathrm{d}}$ and $|\nabla T|$ for both temperature isosurfaces over all turbulence intensity levels. To identify the reason for diminishing $|\nabla T|$, three parameters are identified. These parameters are heat release rate, normal strain rate $\left(K_{\mathrm{N}}\right)$ and curvature $(\kappa)$. Heat release rate is identified with the chemical kinetic effect, whereas $K_{\mathrm{N}}$ and $\kappa$ are the kinematic (due to fluid mechanics) effects. For $665 \mathrm{~K}$ isosurface, $|\nabla T|$ is related to the kinematic effect due to pure curvature. The decrease in $|\nabla T|$ is caused by an increase in magnitude of curvature in the negative direction, which results in a relative increase in the upstream mass flux, thereby increasing $S_{\mathrm{d}}$. For the $1321 \mathrm{~K}$ temperature isosurface, $|\nabla T|$ varies by both curvature and chemical kinetic effects of heat release rate. The decrease in the heat release rate allows dominance of turbulent mixing and homogenization of the gradients.

The time evolution of principal curvatures is examined in order to understand the geometry of the flame islands formed. For both the temperature isosurfaces considered, the study reveal that for all cases of turbulence intensities, the flame islands formed are statistically of ellipsoidal geometry.

Turbulence-chemistry interaction investigated in the Lagrangian viewpoint, accessible through FPT, culminates by the strong correlations between $\left\langle S_{d} / S_{L, T}\right\rangle$ and $\left\langle K a_{S}\right\rangle$ for all 
parametric conditions and different choices of the temperature isosurfaces. A mildly nonlinear behavior was also found, similar to but at much lower degree compared to the laminar flame speed response to steady state stretch. Further studies are needed to develop a general modeling framework for closure of turbulent combustion models based on the Lagrangian flame particle approach.

\section{Acknowledgments}

Work presented in this paper was sponsored by the ISRO-IISc Space Technology Cell, code number ISTC/MAE/SC/334, and competitive research funding from King Abdullah University of Science and Technology (KAUST). The simulations for Case 2 were run on the facility at KAUST Supercomputing Laboratory. We thank Dr. S. Hemchandra for providing the Linux cluster for performing the DNS for Cases 1, 3 and 4 reported in this paper. We would also like to thank Prof. K. N. Lakshmisha for his helpful comments.

\section{References}

[1] N. Peters, Turbulent combustion,, Cambridge university press, 2000.

[2] J.H. Chen, H.G. Im, Proceedings of the Combustion Institute, 28 (2000) 211-218.

[3] J.H. Chen, H.G. Im, Correlation of flame speed with stretch in turbulent premixed methane/air flames, in: Symposium (International) on Combustion, Elsevier, 1998, pp. 819826.

[4] T. Echekki, J.H. Chen, Combustion and Flame, 106 (1996) 184-202.

[5] N. Chakraborty, R. Cant, Physics of Fluids (1994-present), 17 (2005) 105105.

[6] N. Chakraborty, N. Swaminathan, Physics of Fluids (1994-present), 19 (2007) 045104.

[7] L. Cifuentes, C. Dopazo, J. Martin, C. Jimenez, Physics of Fluids (1994-present), 26 (2014) 065108.

[8] C. Dopazo, L. Cifuentes, J. Martin, C. Jimenez, Combustion and flame, (2014).

[9] O. Chatakonda, E.R. Hawkes, A.J. Aspden, A.R. Kerstein, H. Kolla, J.H. Chen, Combustion and flame, 160 (2013) 2422-2433.

[10] S. Lapointe, B. Savard, G. Blanquart, Combustion and flame, 162 (2015) 3341-3355.

[11] B. Savard, G. Blanquart, Combustion and flame, 162 (2015) 2020-2033.

[12] B. Savard, B. Bobbitt, G. Blanquart, Proceedings of the Combustion Institute, 35 (2015) 1377-1384.

[13] Y. Shim, S. Tanaka, M. Tanahashi, T. Miyauchi, Proceedings of the Combustion Institute, 33 (2011) 1455-1462.

[14] J.H. Chen, T. Echekki, W. Kollmann, Combustion and flame, 116 (1999) 15-48.

[15] F. Toschi, E. Bodenschatz, Annual review of fluid mechanics, 41 (2009) 375-404.

[16] P. Yeung, Journal of Fluid Mechanics, 427 (2001) 241-274.

[17] P. Yeung, Annual review of fluid mechanics, 34 (2002) 115-142.

[18] S. Pope, International journal of engineering science, 26 (1988) 445-469.

[19] S. Chaudhuri, Proceedings of the Combustion Institute, 35 (2015) 1305-1312.

[20] G. Lodato, P. Domingo, L. Vervisch, Journal of computational physics, 227 (2008) 5105-5143.

[21] T.J. Poinsot, S. Lele, Journal of computational physics, 101 (1992) 104-129.

[22] C.S. Yoo, H.G. Im, Combustion Theory and Modelling, 11 (2007) 259-286. 
[23] N. Chakraborty, H. Kolla, R. Sankaran, E.R. Hawkes, J.H. Chen, N. Swaminathan, Proceedings of the Combustion Institute, 34 (2013) 1151-1162.

[24] N. Babkovskaia, N. Haugen, A. Brandenburg, Journal of computational physics, 230 (2011) 1-12.

[25] J. Li, Z. Zhao, A. Kazakov, F.L. Dryer, Int J Chem Kinet, 36 (2004) 566-575.

[26] P. Arias, H. Im, P. Narayanan, A. Trouvé, Proceedings of the Combustion Institute, 33 (2011) 2591-2597.

[27] M.P. Burke, M. Chaos, Y. Ju, F.L. Dryer, S.J. Klippenstein, Int J Chem Kinet, 44 (2012) 444-474.

[28] T. Möller, B. Trumbore, Journal of graphics tools, 2 (1997) 21-28.

[29] S. Chaudhuri, Physical Review E, 91 (2015) 021001.

[30] C.K. Law, Combustion physics, Cambridge University Press, 2006.

[31] R.J. Kee, F.M. Rupley, J.A. Miller, Chemkin-II: A Fortran chemical kinetics package for the analysis of gas-phase chemical kinetics, in, Sandia National Labs., Livermore, CA (USA), 1989.

[32] N. Swaminathan, R.W. Grout, Physics of Fluids, 18 (2006) 045102.

[33] N. Chakraborty, N. Swaminathan, Physics of Fluids, 19 (2007) 045103.

[34] A.N. Lipatnikov, J. Chomiak, V.A. Sabelnikov, S. Nishiki, T. Hasegawa, Proceedings of the Combustion Institute, 35 (2015) 1401-1408.

[35] N. Fogla, F. Creta, M. Matalon, Combustion and flame, (2015).

[36] A.Y. Poludnenko, E.S. Oran, Combustion and flame, 158 (2011) 301-326.

[37] C. Altantzis, C.E. Frouzakis, A.G. Tomboulides, K. Boulouchos, Combustion and flame, 162 (2015) 331-344.

[38] J.H. Chen, E.R. Hawkes, R. Sankaran, S.D. Mason, H.G. Im, Combustion and flame, 145 (2006) 128-144.

[39] S. Chaudhuri, V.y. Akkerman, C.K. Law, Physical Review E, 84 (2011) 026322.

[40] J. Bechtold, M. Matalon, Combustion and flame, 127 (2001) 1906-1913.

[41] D. Bradley, P. Gaskell, X. Gu, Combustion and flame, 104 (1996) 176-198.

[42] P. Clavin, J.C. Graña-Otero, Journal of Fluid Mechanics, 686 (2011) 187-217.

[43] A. Trouvé, T. Poinsot, Journal of Fluid Mechanics, 278 (1994) 1-31.

[44] S. Pope, W. Cheng, The stochastic flamelet model of turbulent premixed combustion, in: Symposium (International) on Combustion, Elsevier, 1989, pp. 781-789. 


\section{Nomenclature}

A Pre-exponential factor

C Concentration of species

$c_{\mathrm{p}}$ Constant pressure specific heat

$D$ Mass diffusivity

$h$ Specific enthalpy

$k$ Specific reaction constant

$K a$ Turbulent Karlovitz number

$$
=\left(\frac{S_{L, O} \delta_{L}}{v_{0}}\right)^{1 / 2}\left(\frac{u_{r m s}}{S_{L, 0}}\right)^{3 / 2}\left(\frac{l_{I}}{\delta_{L}}\right)^{-1 / 2}
$$

$K a_{s}$ Stretch Karlovitz number

$K a_{T}$ Tangential component of stretch Karlovitz number

$K a_{C}$ Curvature component of stretch Karlovitz number

Le Lewis number

$L e_{H 2}$ Lewis number based on the deficient species

$L_{M}$ Markstein length

$l_{\mathrm{I}} \quad$ Integral length scale

n Unit normal vector to the flame surface

$p \quad$ Thermodynamic pressure

\section{Greek Symbols}

$v$ Kinematic viscosity

$v^{\prime}$ Stoichiometric coefficient of the reactant species

$v^{\prime \prime}$ Stoichiometric coefficient of the product species

$\rho$ Density of the mixture

$\lambda$ Thermal conductivity of the mixture

$\dot{\omega}^{\prime \prime \prime}$ Production/consumption rate of species

$\phi$ Equivalence ratio

$\delta_{L} \quad$ Thermal thickness of flame $=\left(T_{b}-T_{u}\right) /|\nabla T|_{\max }$

\section{Subscripts}

$\alpha$ Species index

f Forward reaction

r Backward reaction

u Unburnt mixture

b Burnt mixture

\section{Superscript}

$\beta$ Temperature exponent
Da Damköhler Number

$=S_{L, 0} l_{I} / u_{r m s} \delta_{L}$

$\mathrm{E}_{\mathrm{a}}$ Activation Energy

$f$ mass flux

$\mathrm{R}_{\mathrm{u}} \quad$ Universal gas constant

Re Reynolds number

$R e_{\mathrm{T}}$ Turbulent Reynolds number

$=u_{r m s} l_{I} / v_{0}$

$S_{L, T}$ Planar laminar flame speed relative to the local flow at a given local temperature condition

$S_{\mathrm{d}} \quad$ Displacement flame speed

$S_{11}^{*}$ Tangential component of strain rate along principal direction

$T$ Temperature

$t$ Time

$\langle\mathbf{U}\rangle$ Mean velocity

$\mathrm{U}_{3}$ Flow velocity along local flame normal

$u_{r m s}$ RMS of the fluctuating velocity

$y_{1}^{*}$ Coordinate in the principal direction

$Y \quad$ Species mass fraction

$\eta$ Kolmogorov length scale

$\tau_{\text {track }}$ Tracking time

$\tau_{I}$ Integral time scale

$\tau_{\eta}$ Kolmogorov time scale

$\tau_{F, L}$ Lifetime of flame particle

$\langle\psi\rangle$ Ensemble average of variable $\psi$

$\Sigma^{\prime}$ Flame surface area per unit volume in Inlet condition 
Tables and Figures:

\begin{tabular}{|c|c|c|c|c|}
\hline & Case 1 & Case 2 & Case 3 & Case 4 \\
\hline Grid & $256 \times 128 \times 128$ & $512 \times 256 \times 256$ & $256 \times 128 \times 128$ & $384 \times 128 \times 128$ \\
\hline $\begin{array}{c}\text { Domain (cm) } \\
\mathrm{L}_{x} \times \mathrm{L}_{y} \times \mathrm{L}_{z}\end{array}$ & $1.0 \times 0.5 \times 0.5$ & $2.0 \times 1.0 \times 1.0$ & $1.0 \times 0.5 \times 0.5$ & $1.2 \times 0.4 \times 0.4$ \\
\hline$\delta_{L} /_{\Delta_{D N S}}$ & 9 & 9 & 9 & 12 \\
\hline$\langle\mathbf{U}\rangle(\mathbf{c m} / \mathbf{s})$ & 350 & 680 & 500 & 700 \\
\hline$u_{\mathrm{rms}}(\mathrm{cm} / \mathrm{s})$ & 192 & 678 & 503 & 557 \\
\hline $\boldsymbol{R e}_{T}$ & 146 & 1623 & 351 & 198 \\
\hline Ka & 3.77 & 14.4 & 16.7 & 20.22 \\
\hline$D a$ & 3.26 & 5 & 1.1 & 0.68 \\
\hline$L e_{H 2}$ & 0.84 & 0.62 & 0.84 & 0.84 \\
\hline$l_{I}(\mathrm{~cm})$ & 0.119 & 0.5 & 0.109 & 0.074 \\
\hline$\tau_{I}(\boldsymbol{\mu} \mathbf{s})$ & 627 & 731 & 217 & 133 \\
\hline $\boldsymbol{\eta}(\boldsymbol{\mu} \mathbf{m})$ & 28 & 19.4 & 13 & 15 \\
\hline $\boldsymbol{\tau}_{\boldsymbol{\eta}}(\boldsymbol{\mu} \mathbf{s})$ & 52 & 18.2 & 12 & 10 \\
\hline$T_{\text {in }}(\mathbf{K})$ & 310 & 300 & 310 & 310 \\
\hline$p_{\text {in }}(\operatorname{atm})$ & 1.0 & 1.0 & 1.0 & 1.0 \\
\hline$\phi$ & 0.81 & 0.7 & 0.81 & 0.81 \\
\hline$\delta_{\mathrm{L}}(\mu \mathrm{m})$ & 361 & 354 & 361 & 361 \\
\hline$\Delta \tau_{\text {track }}(\mu \mathbf{s})$ & 4 & 10 & 2.5 & 2.5 \\
\hline$\tau_{\text {track }}(\mu \mathbf{s})$ & 680 & 990 & 292 & 208 \\
\hline
\end{tabular}

Table 1. List of simulation parameters

\begin{tabular}{|c|c|c|}
\hline $\boldsymbol{T}$ & $\mathbf{6 6 5} \mathbf{K}$ & $\mathbf{1 3 2 1} \mathbf{~ K}$ \\
\hline$S_{\mathrm{d}}$ Analytical $(\mathrm{cm} / \mathrm{s})$ & 379.22 & 741 \\
\hline$S_{L, T}$ CHEMKIN $(\mathrm{cm} / \mathrm{s})$ & 372 & 713.4 \\
\hline
\end{tabular}

Table 2. Displacement flame speed $\left(S_{\mathrm{d}}\right)$ validation 


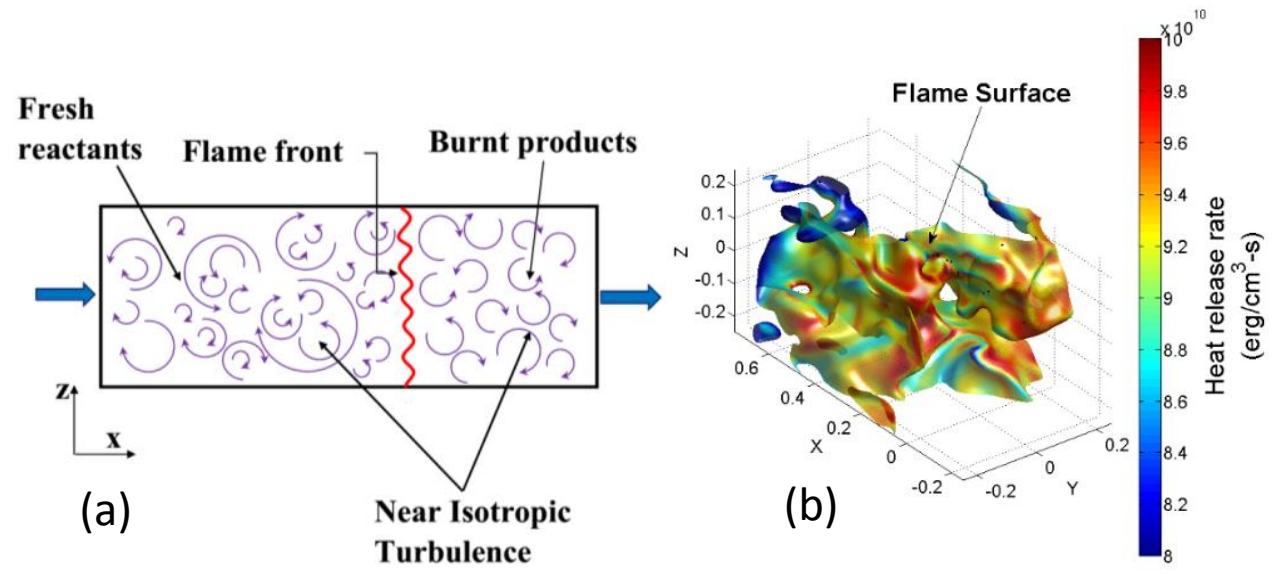

Figure 1. (a) Schematic of the computational domain (mean flow is in the positive $x$-direction whereas flame propagates in the negative $\mathrm{x}$-direction). (b) Schematic of the 3D domain showing $1321 \mathrm{~K}$ temperature isosurface from Case 3.

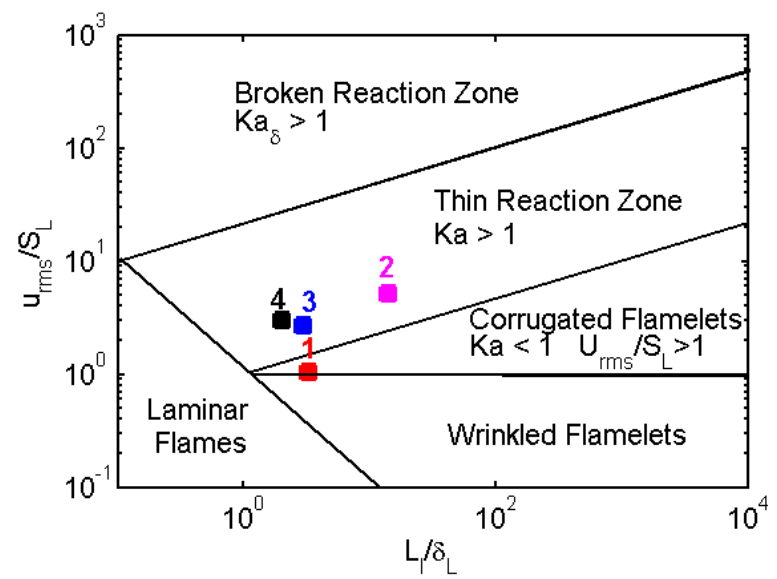

Figure 2. Regime diagram and the simulation conditions.

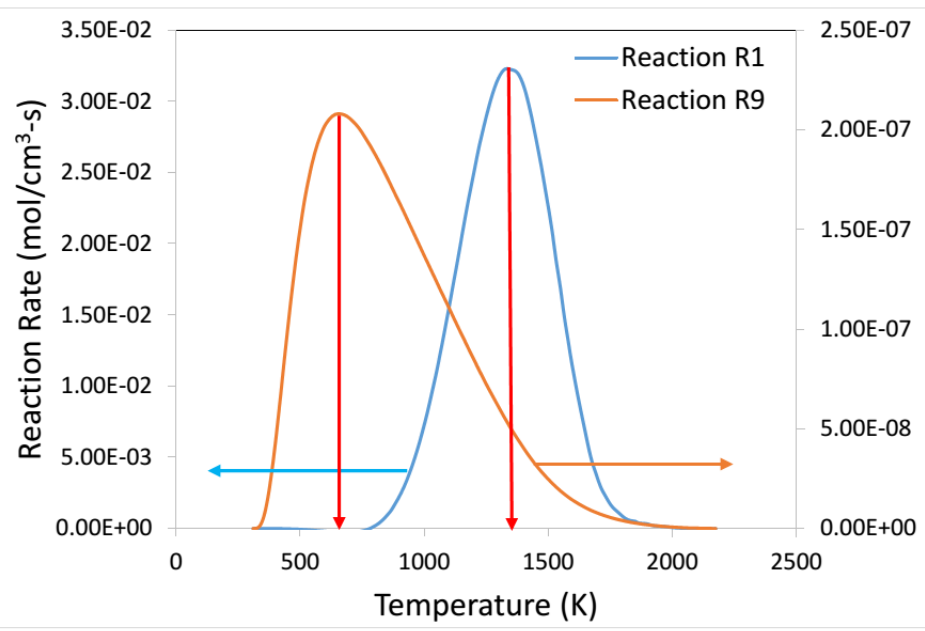

Figure 3. Reaction rates for R1 and $\mathrm{R} 9$ as a function of temperature for the laminar flame at the equivalence ratio of 0.81 . 

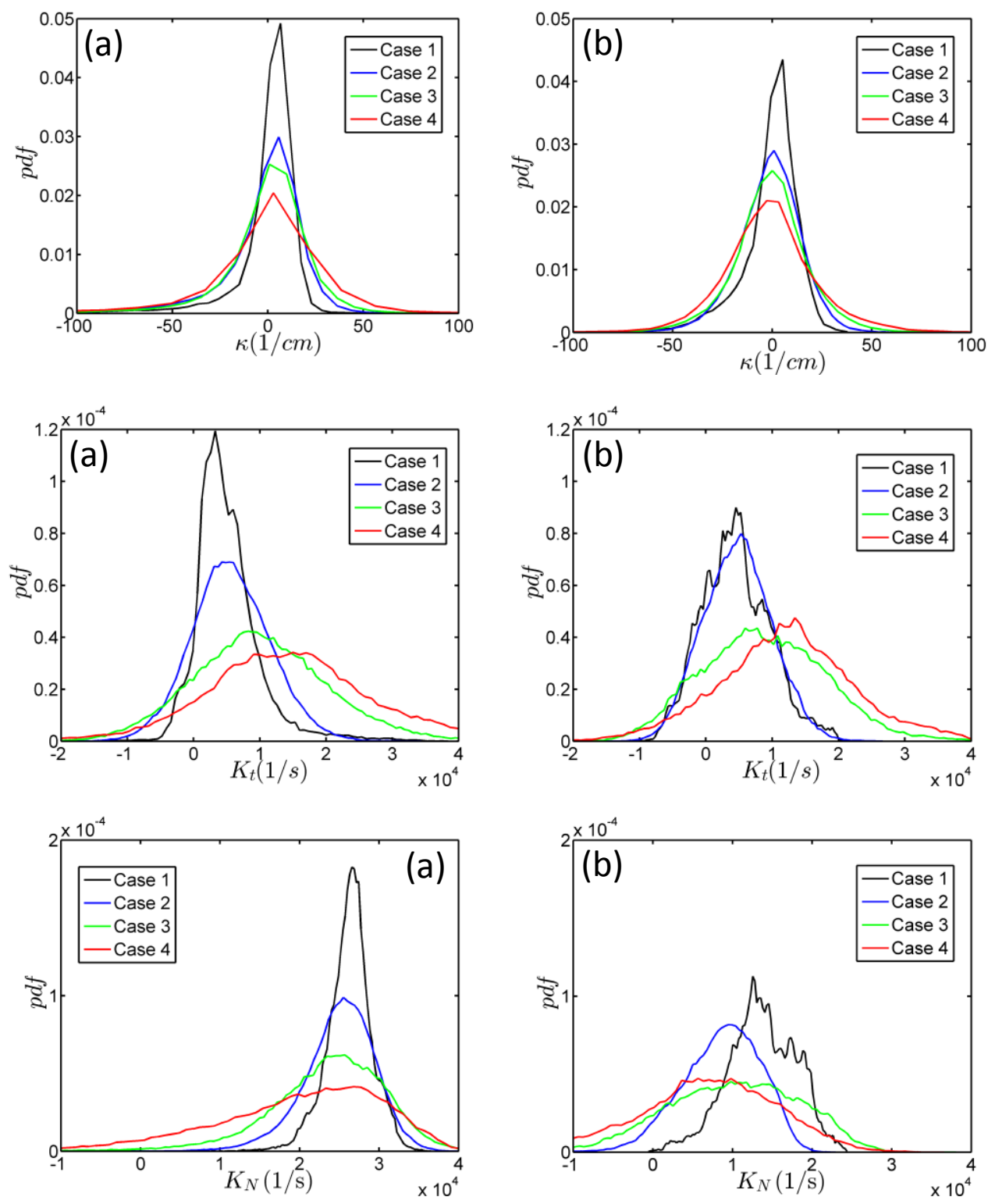

Figure 4. Probability density function (pdf) of curvature ( $\kappa$, top), tangential strain rate $\left(K_{\mathrm{t}}\right.$, middle), and normal strain rate $\left(K_{\mathrm{N}}\right.$, bottom) for the temperature isosurface at $t=\tau_{\text {track }}$ (a) $T=665 \mathrm{~K}$ (b) $T=1321$ K. 

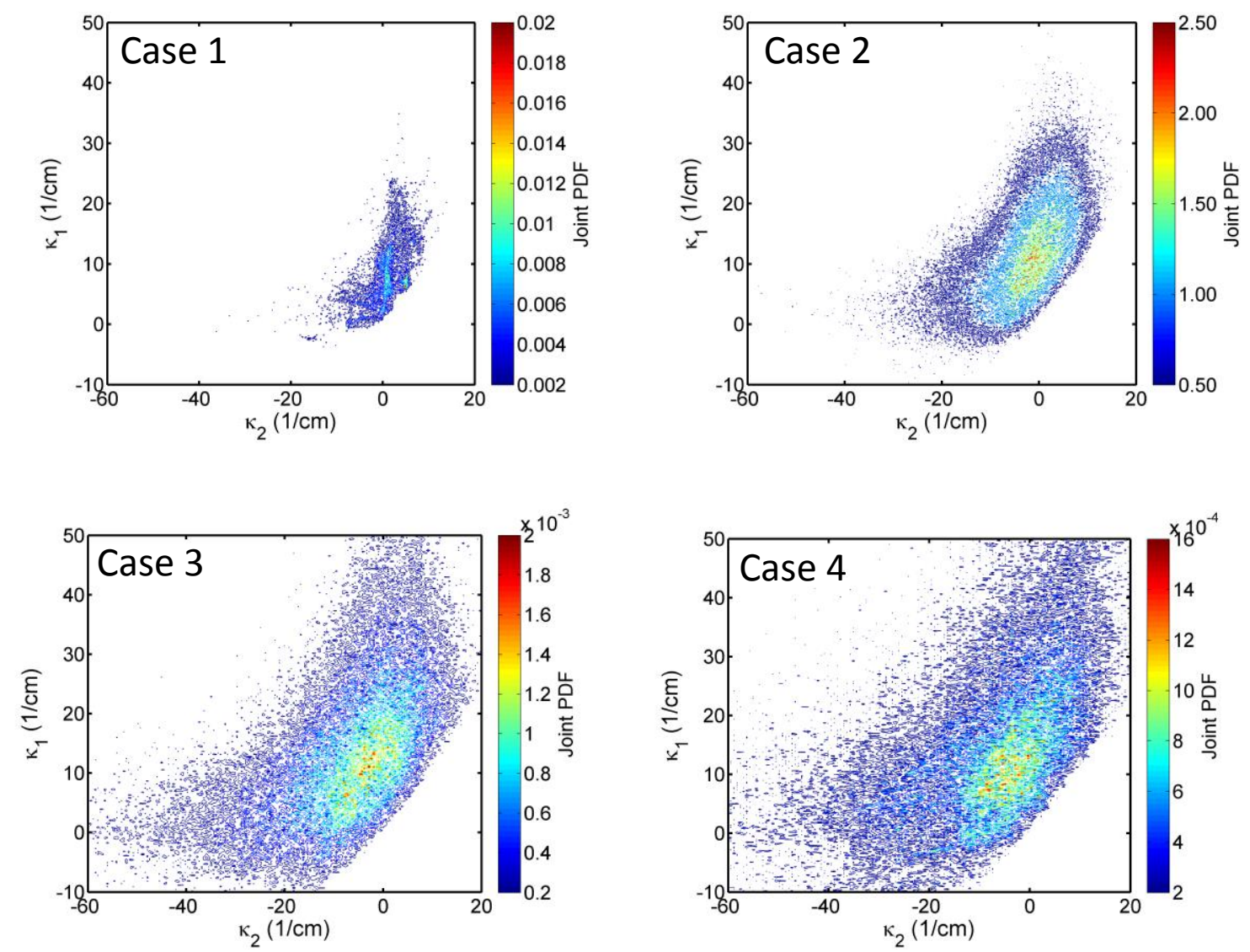

Figure 5. Joint probability density function of principal curvatures $\left(\kappa_{1}\right.$ and $\left.\kappa_{2}, \kappa_{1}<\kappa_{2}\right)$ for the entire temperature isosurface for $T=665 \mathrm{~K}$ at $t=\tau_{\text {track }}$ for Cases $1-4$. 

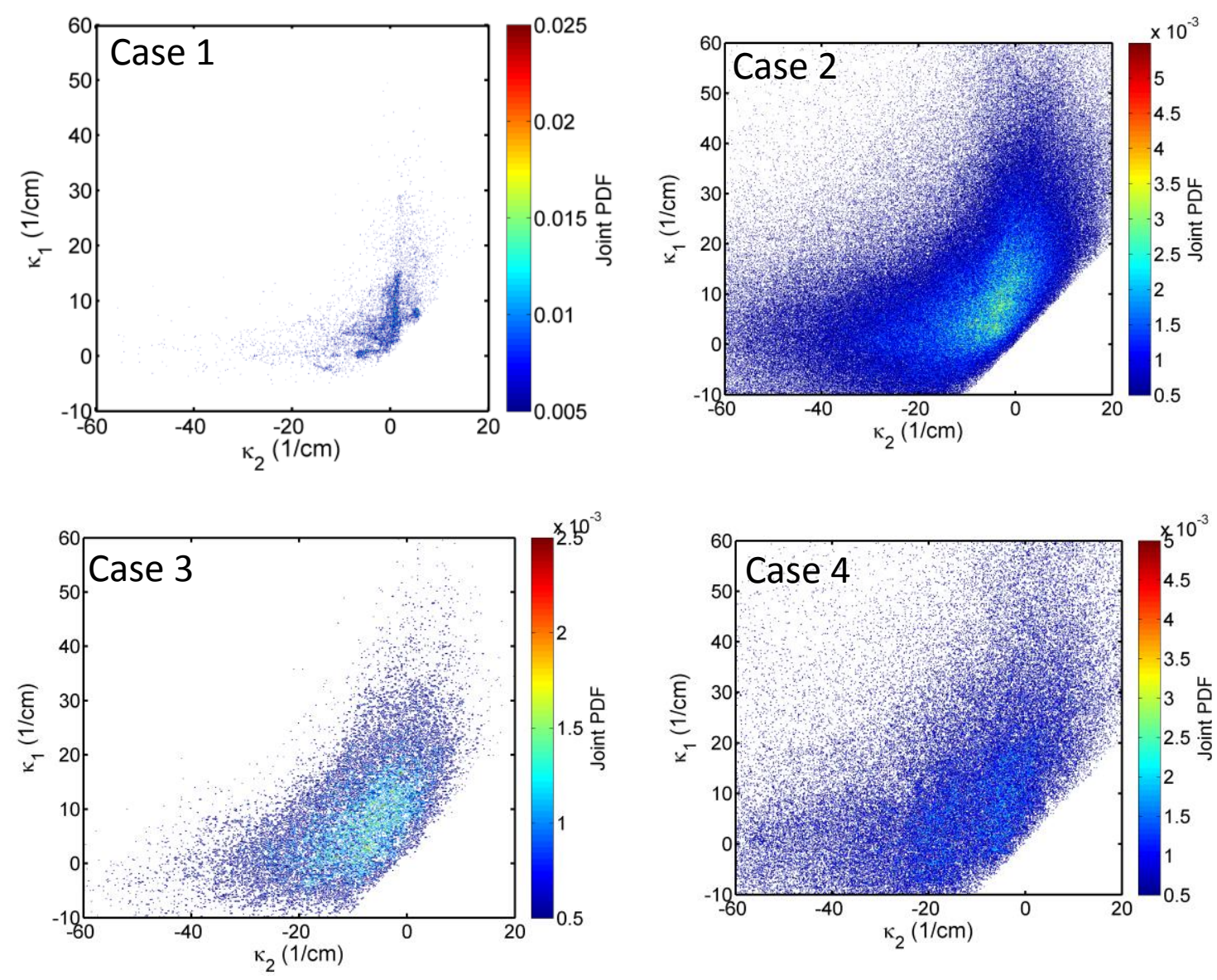

Figure 6. Joint probability density function of principal curvatures $\left(\kappa_{1}\right.$ and $\left.\kappa_{2}, \kappa_{1}<\kappa_{2}\right)$ for the entire temperature isosurface for $T=1321 \mathrm{~K}$ at $t=\tau_{\text {track }}$ for Cases 1-4. 

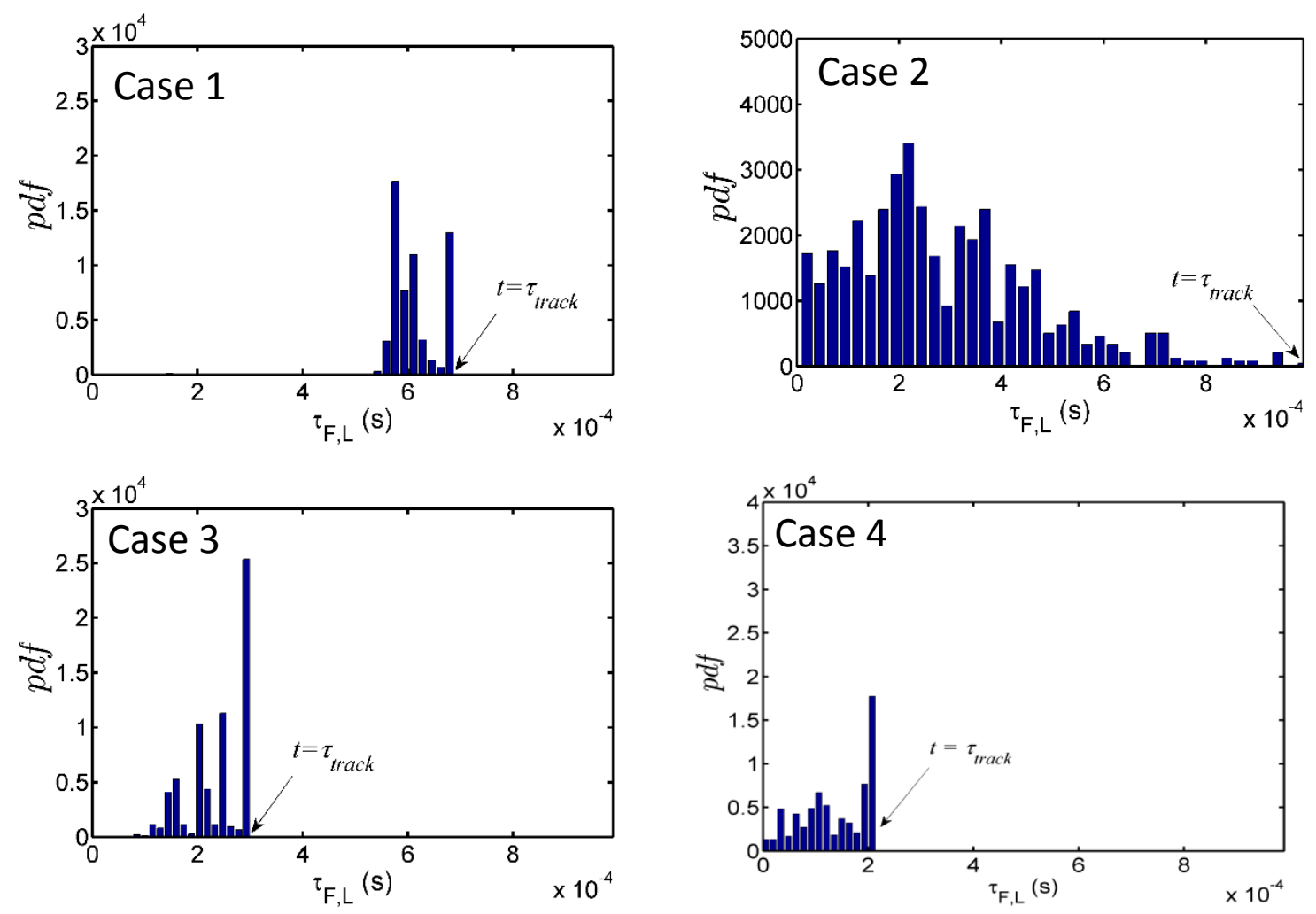

Figure 7. Probability density function (pdf) of flame particle lifetime $\left(\tau_{F, L}\right)$ residing on $665 \mathrm{~K}$ isosurface for Cases 1-4. 

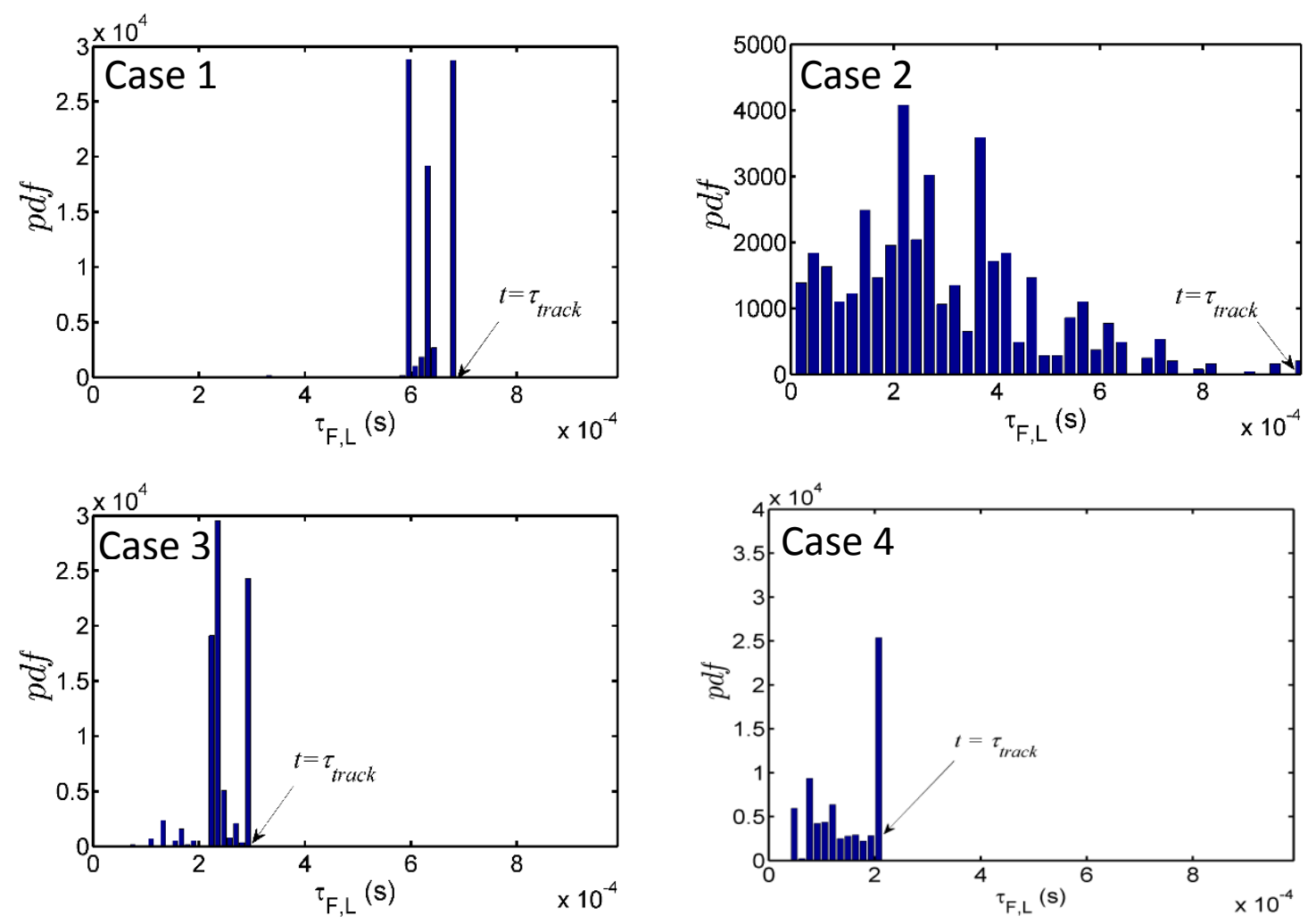

Figure 8. Probability density function (pdf) of flame particle lifetime $\left(\tau_{F, L}\right)$ residing on $1321 \mathrm{~K}$ isosurface for Cases 1-4. 

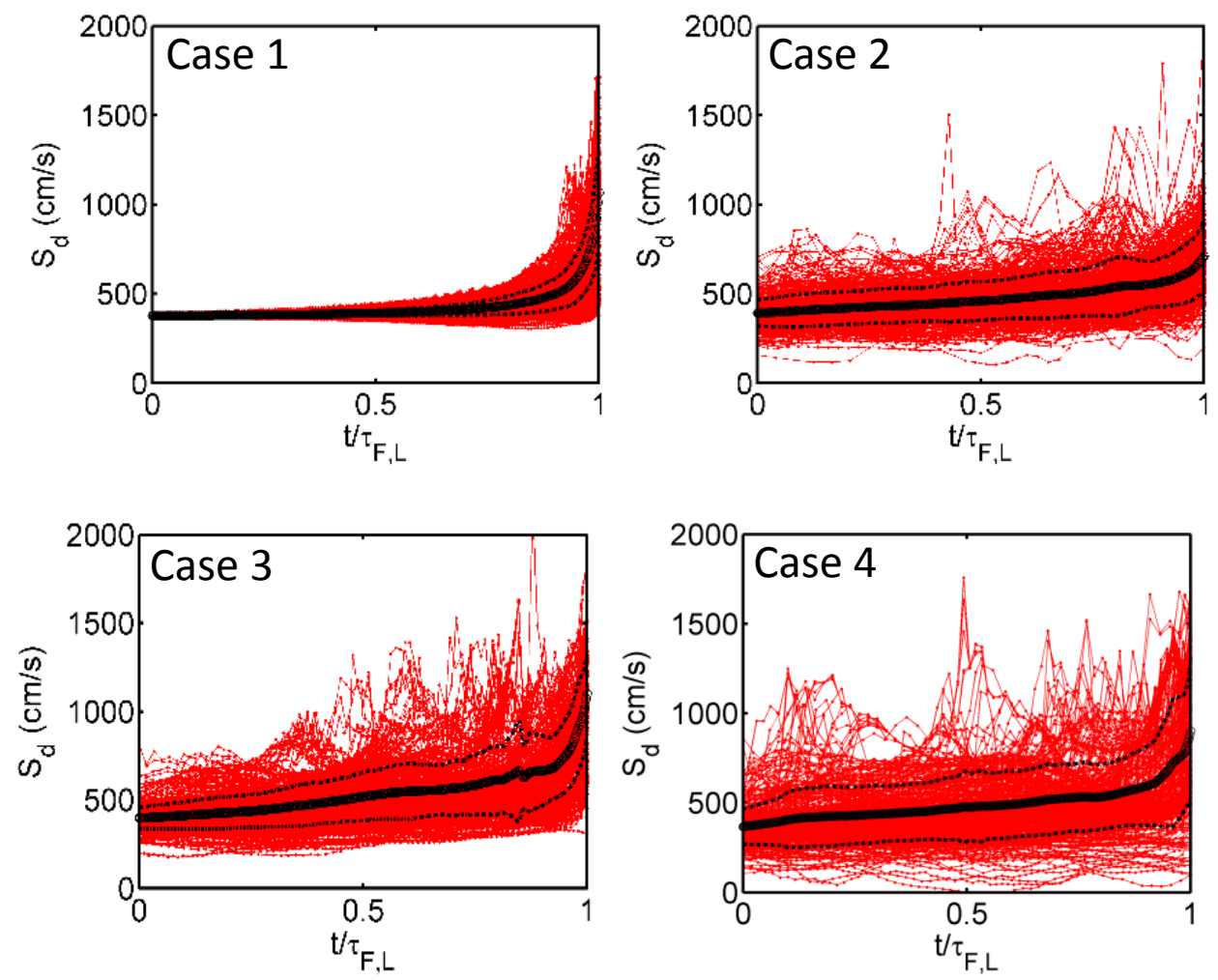

Figure 9. Displacement flame speed $\left(S_{\mathrm{d}}\right)$ vs. normalized time $\left(t / \tau_{F, L}\right)$ for $665 \mathrm{~K}$ isosurface for Cases 1-4. Time evolution of the individual flame particles are shown by red lines and the solid black lines represent the ensemble average over all the particles at a given time instant. Dotted black lines represent the standard deviation of the variables about the mean. The line convention remains the same in all subsequent plots unless otherwise mentioned. 

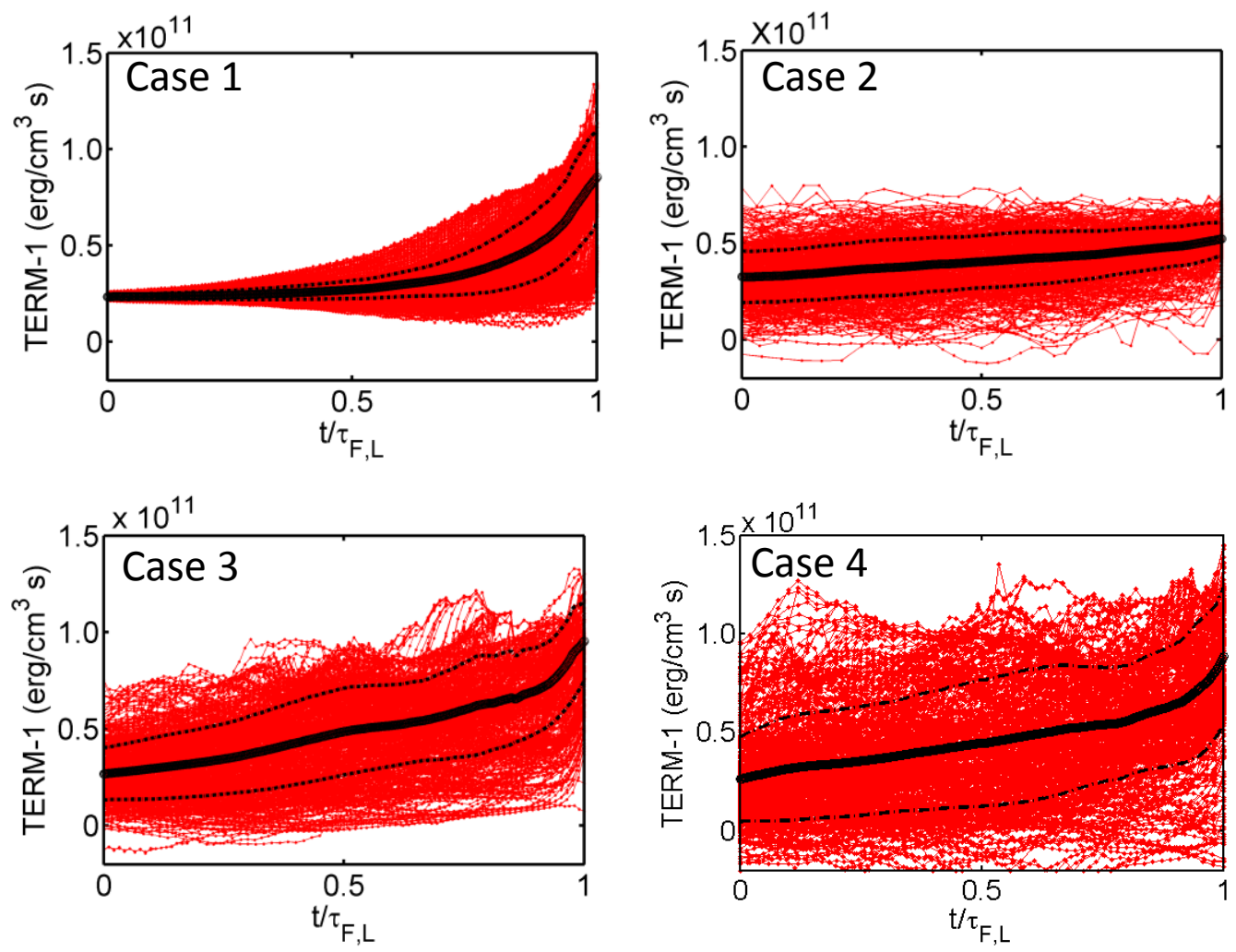

Figure 10. Term 1 of $S_{\mathrm{d}}$ equation vs. normalized time $\left(t / \tau_{F, L}\right)$ for $665 \mathrm{~K}$ isosurface for Cases 1-4.
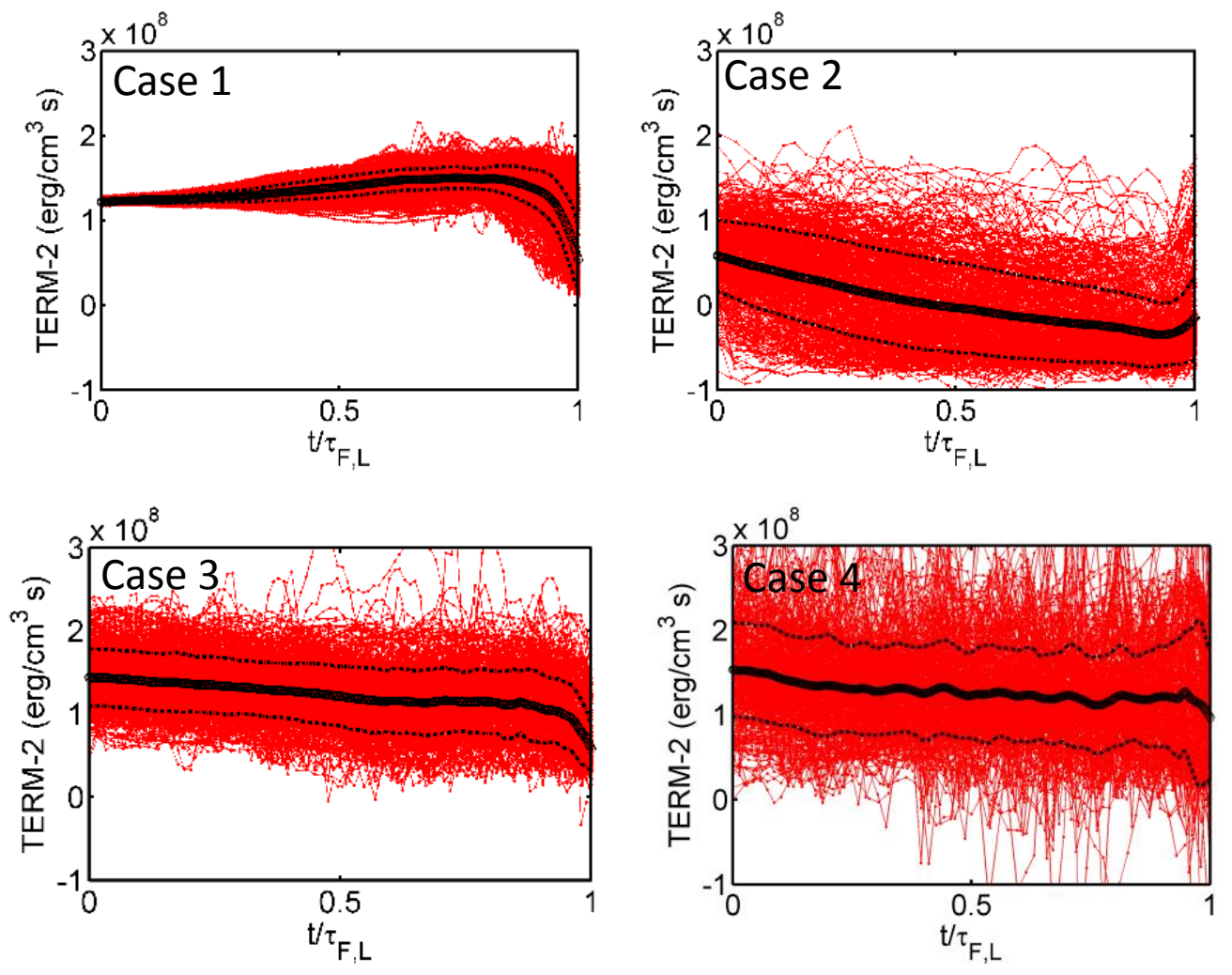

Figure 11. Term 2 of $S_{\mathrm{d}}$ equation vs. normalized time $\left(t / \tau_{F, L}\right)$ for $665 \mathrm{~K}$ isosurface for Cases 1-4. 

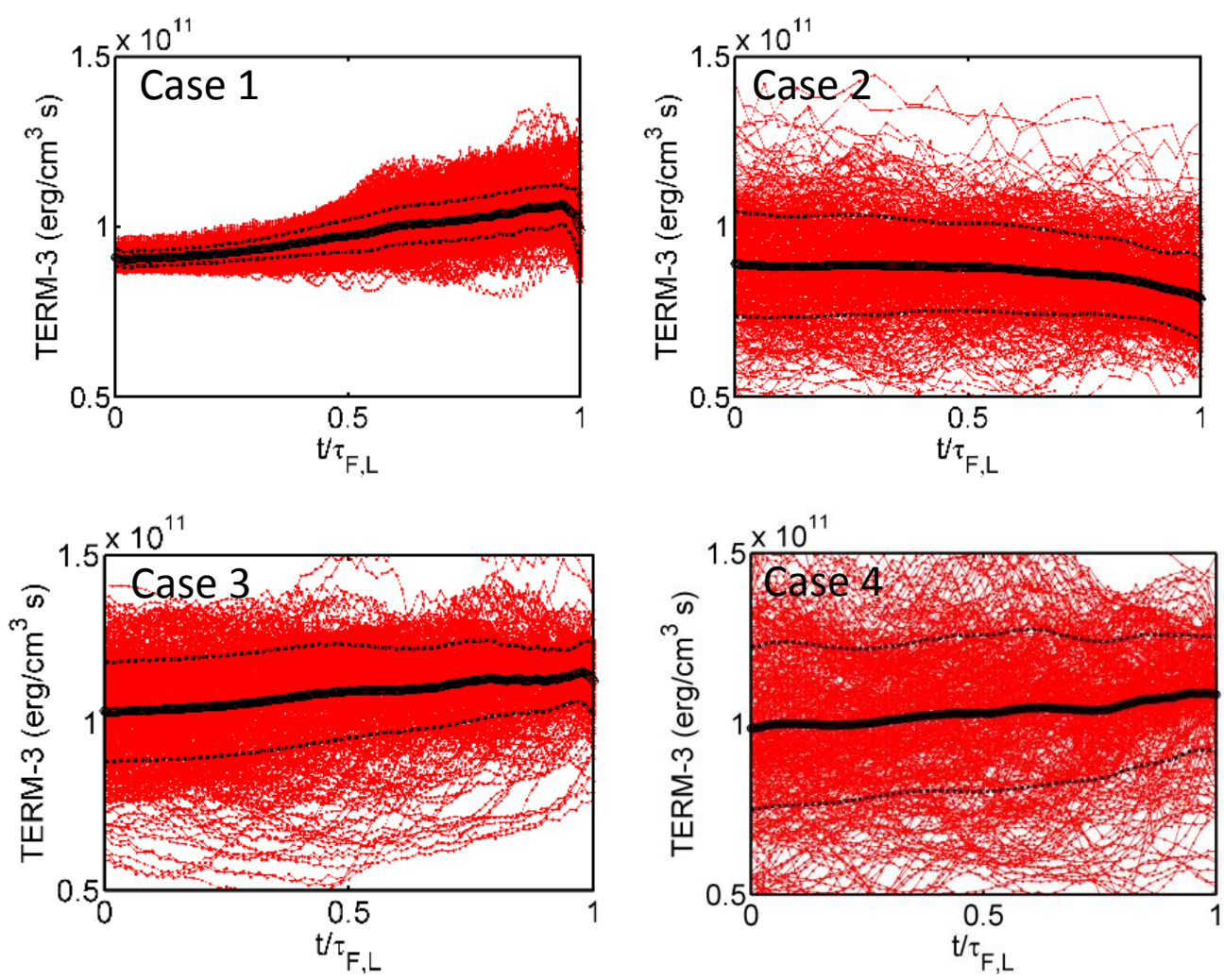

Figure 12. Term 3 of $S_{\mathrm{d}}$ equation vs. normalized time $\left(t / \tau_{F, L}\right)$ for $665 \mathrm{~K}$ isosurface for Cases 1-4.
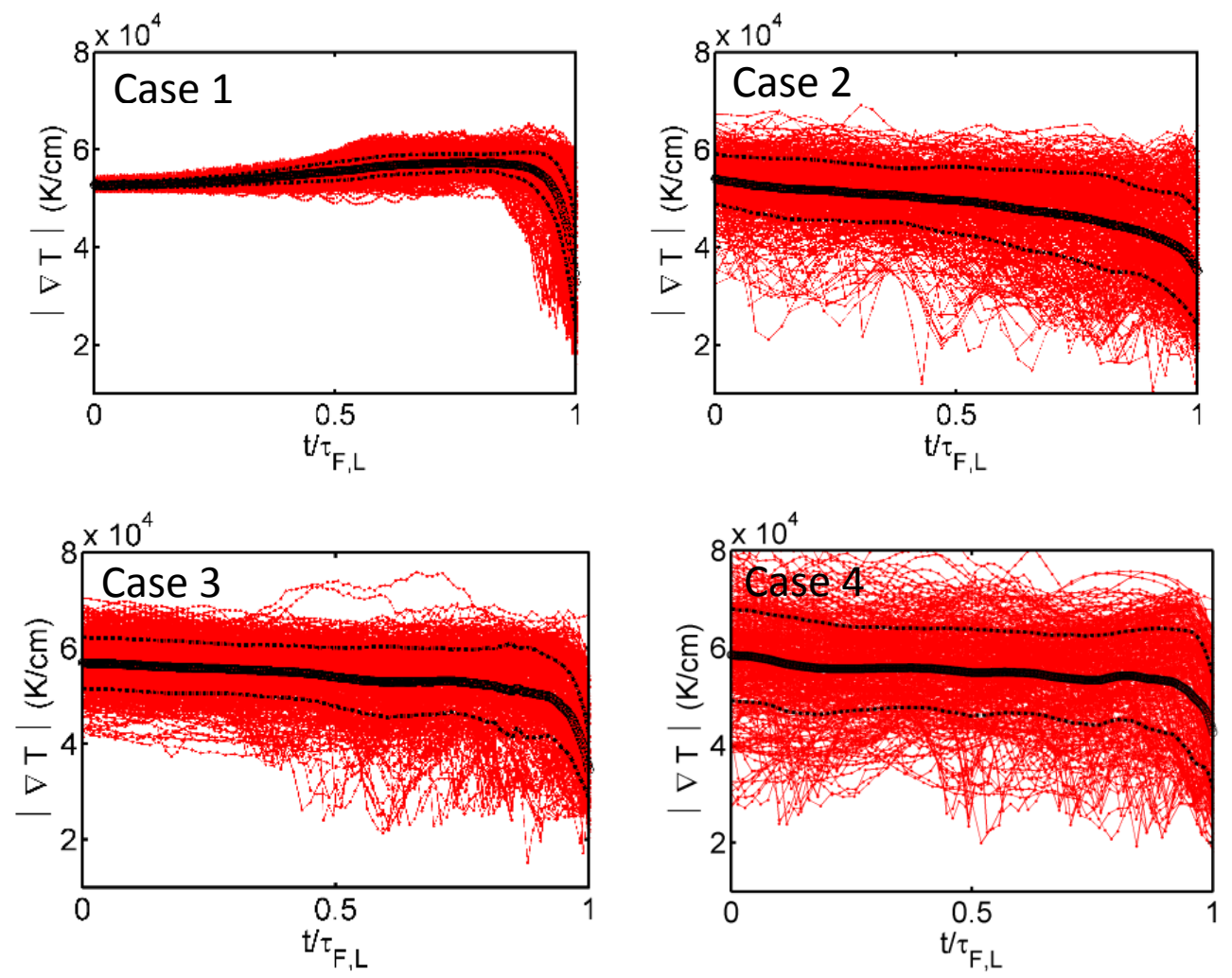

Figure 13. Magnitude of temperature gradient $(|\nabla T|)$ vs. normalized time $\left(t / \tau_{F, L}\right)$ for $665 \mathrm{~K}$ isosurface for Cases 1-4. 

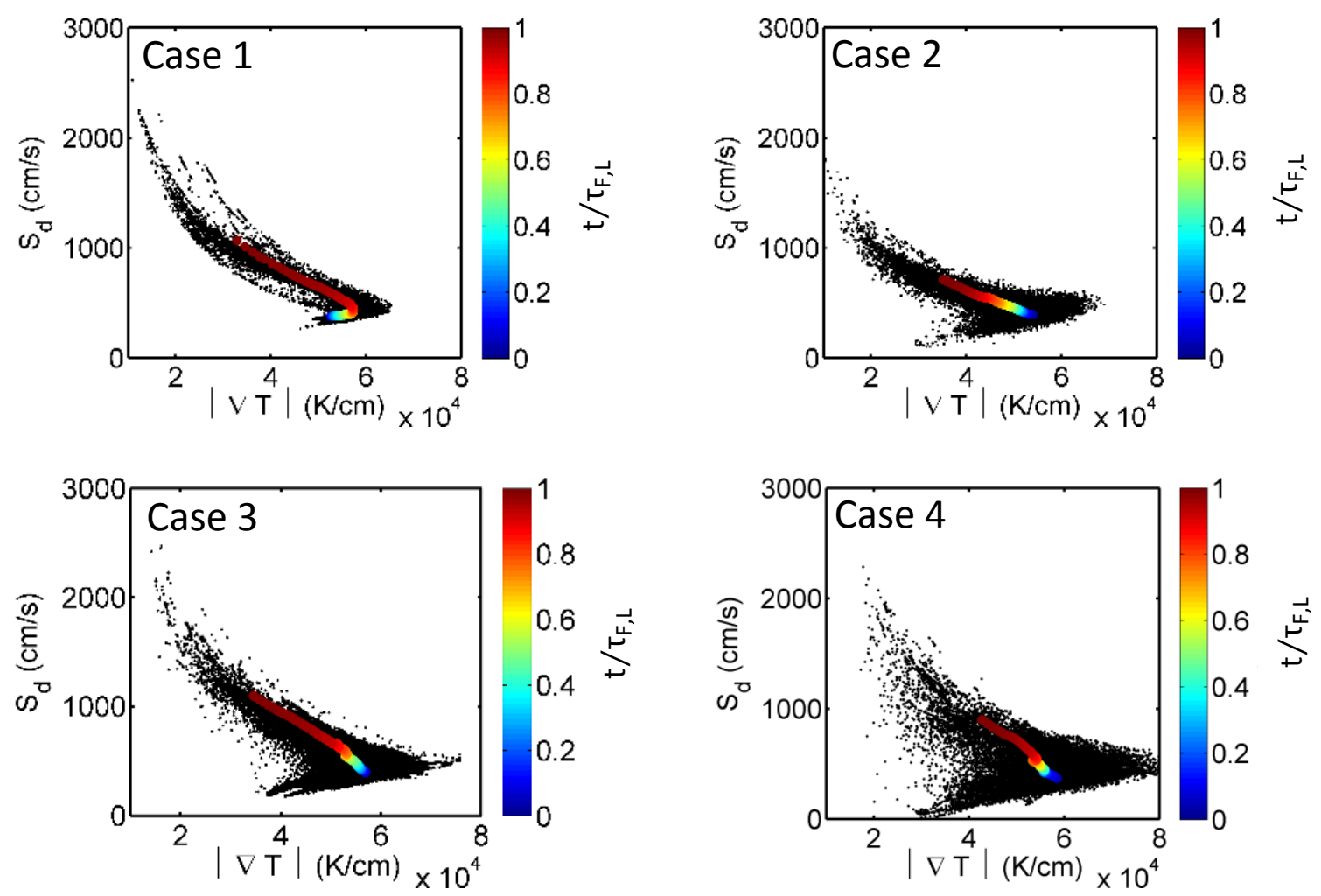

Figure 14. Joint evolution of displacement flame speed $\left(S_{\mathrm{d}}\right)$ and magnitude of gradient $(|\nabla T|)$ with normalized time $\left(t / \tau_{F, L}\right)$ for $665 \mathrm{~K}$ isosurface for Cases 1-4.
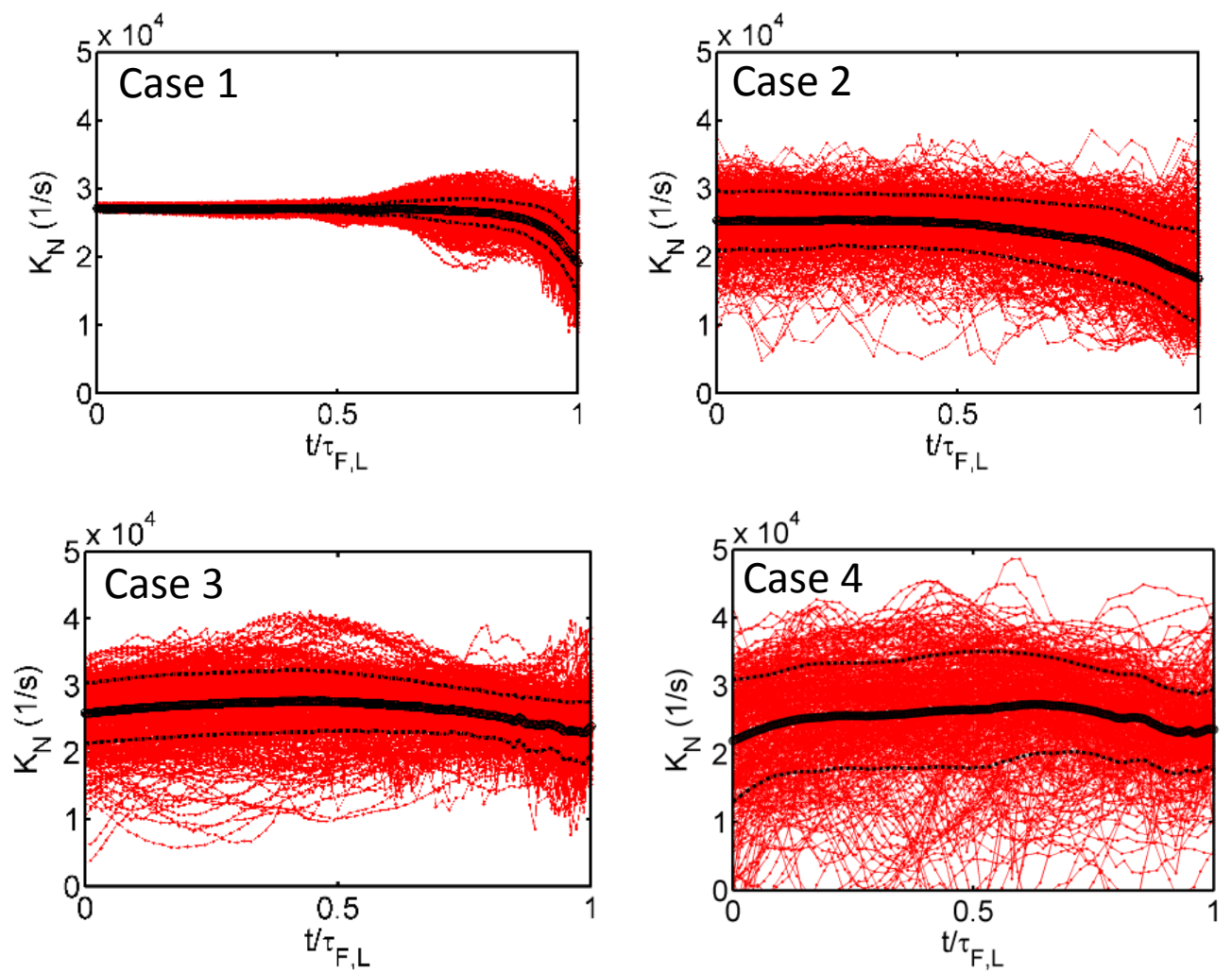

Figure 15. Normal strain rate $\left(K_{\mathrm{N}}\right)$ vs. normalized time $\left(t / \tau_{F, L}\right)$ for $665 \mathrm{~K}$ isosurface for Cases 1-4. 

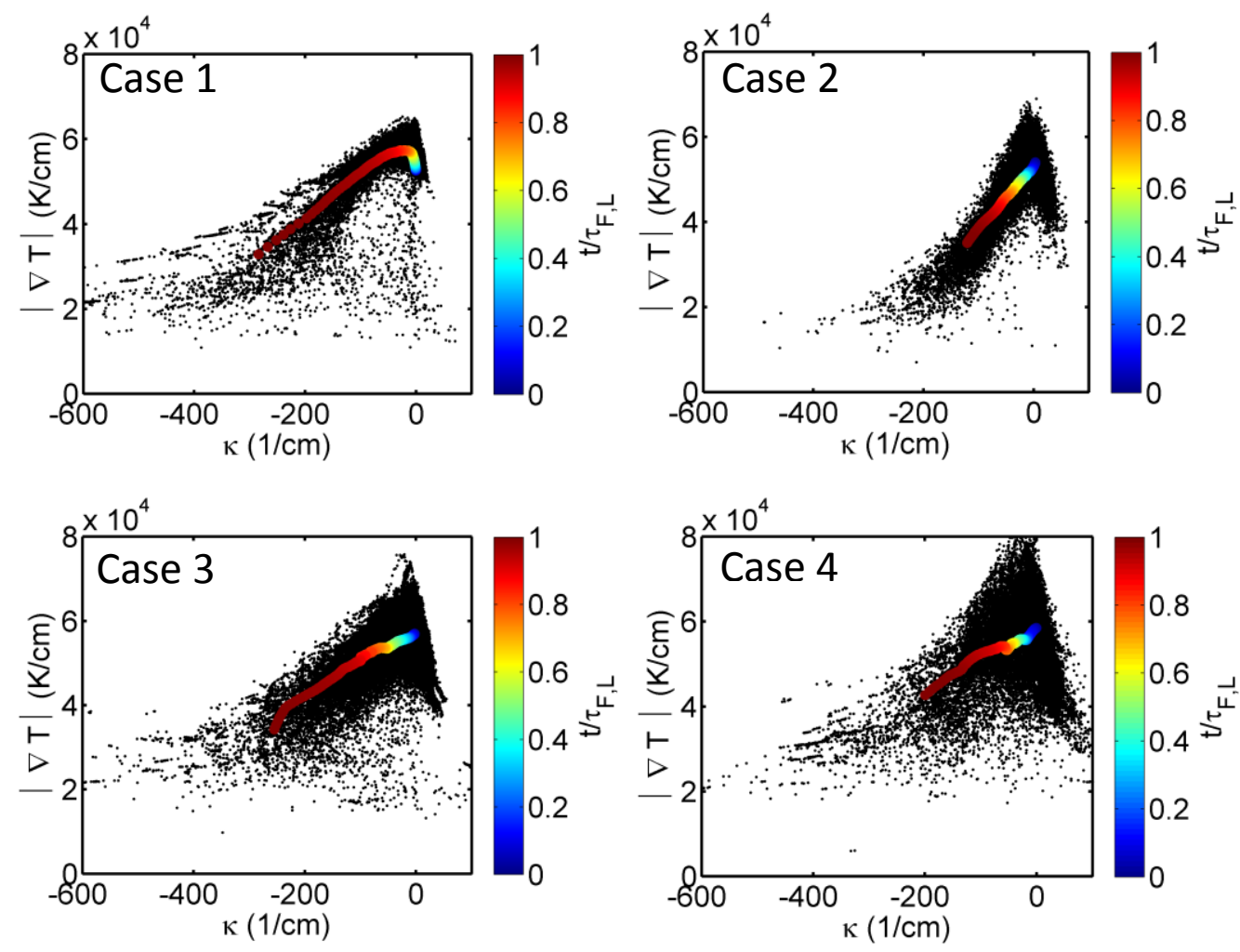

Figure 16. Joint evolution of magnitude of temperature gradient $(|\nabla T|)$ and mean curvature $(\kappa)$ with normalized time $\left(t / \tau_{F, L}\right)$ for $665 \mathrm{~K}$ isosurface for Cases 1-4. 

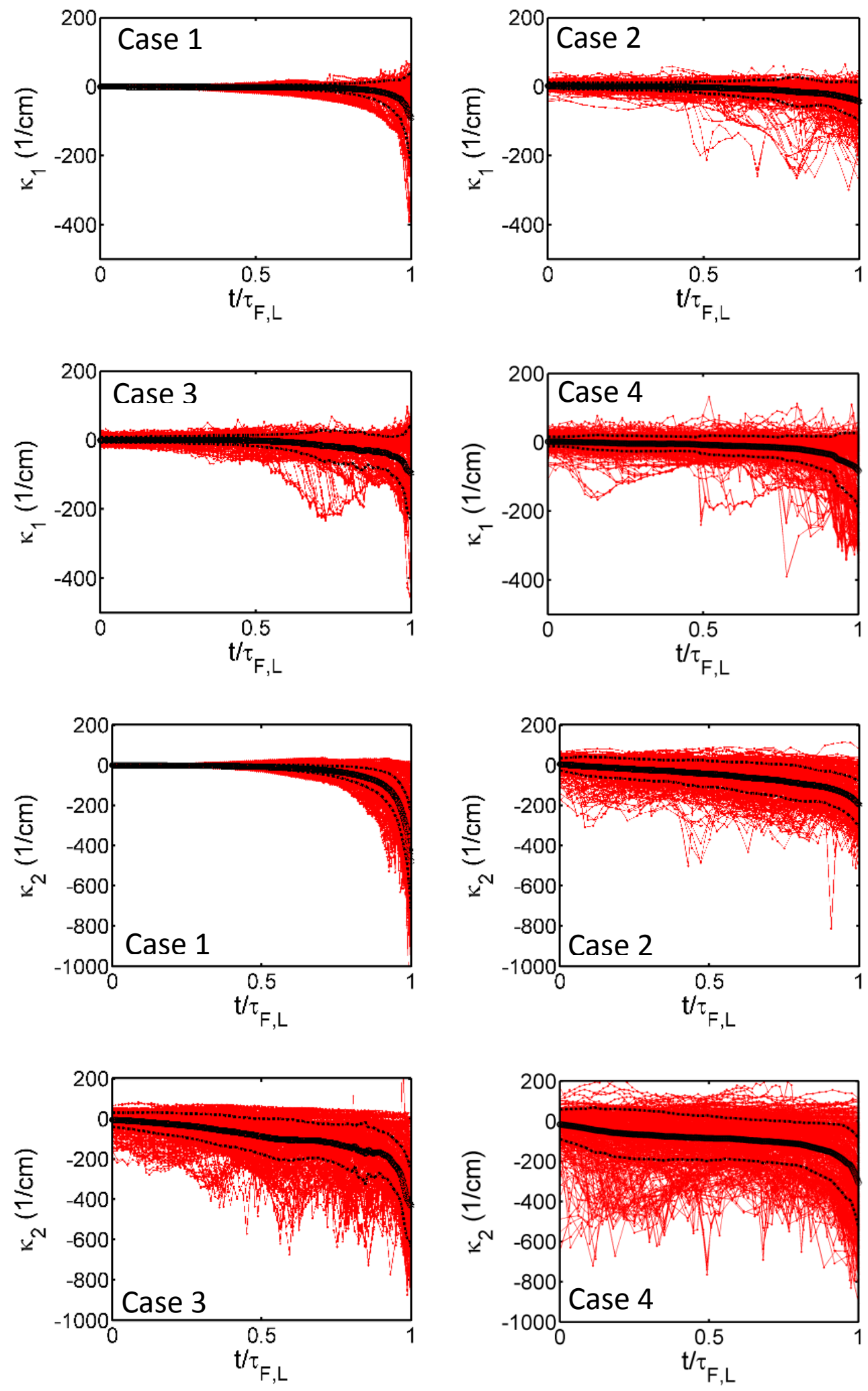

Figure 17. Evolution of principal curvatures, $\kappa_{1}$ (top rows) and $\kappa_{2}$ (bottom rows) $\left(\left|\kappa_{1}\right|<\left|\kappa_{2}\right|\right)$, vs. normalized time $\left(t / \tau_{F, L}\right)$ for $665 \mathrm{~K}$ isosurface for Cases 1-4. 

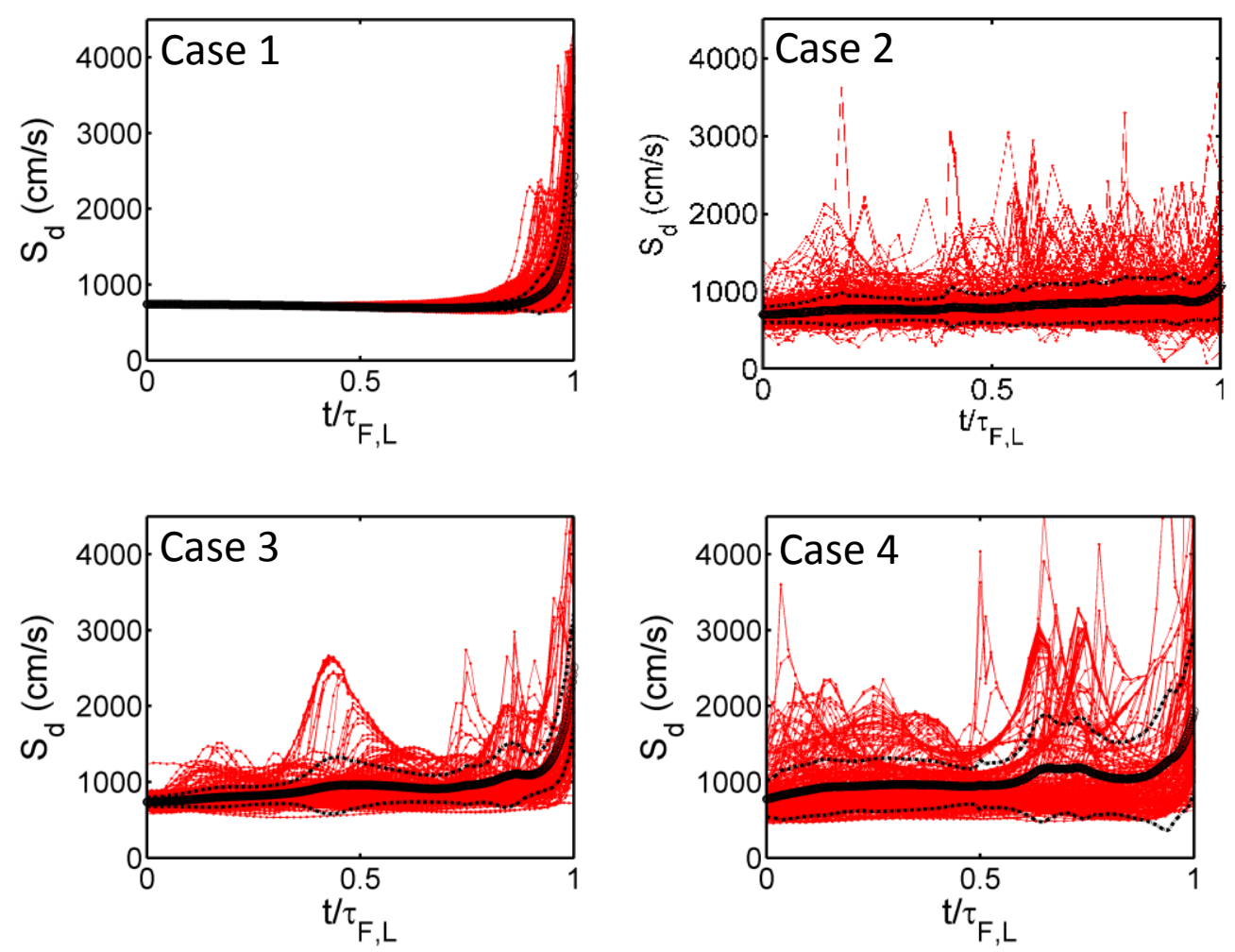

Figure 18. Displacement flame speed $\left(S_{\mathrm{d}}\right)$ vs. normalized time $\left(t / \tau_{F, L}\right)$ for $1321 \mathrm{~K}$ isosurface for Cases 1-4.
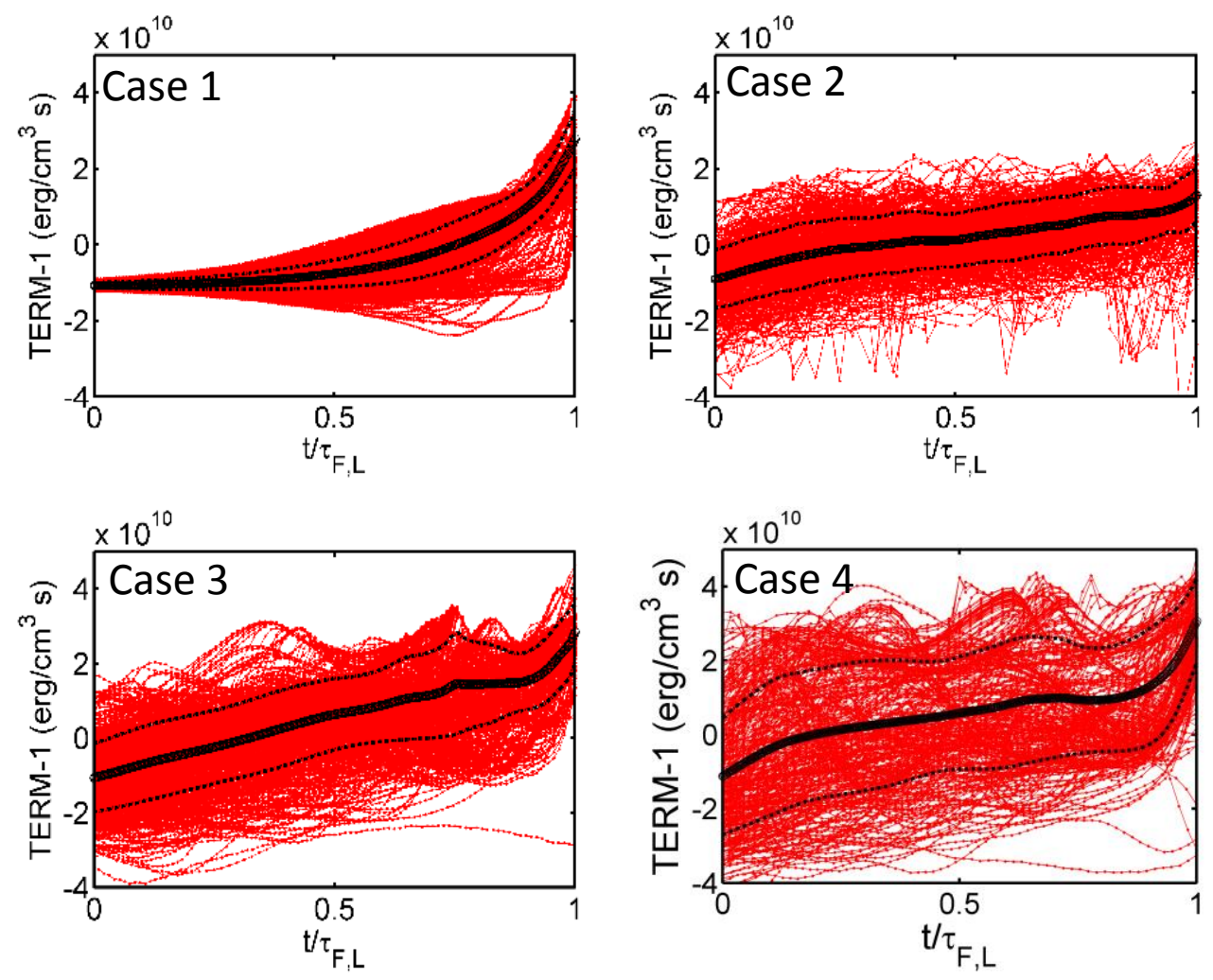

Figure 19. Term 1 of $S_{\mathrm{d}}$ equation vs. normalized time $\left(t / \tau_{F, L}\right)$ for $1321 \mathrm{~K}$ isosurface: (a) Case 1 , (b) Case 2, (c) Case 3 and (d) Case 4. 

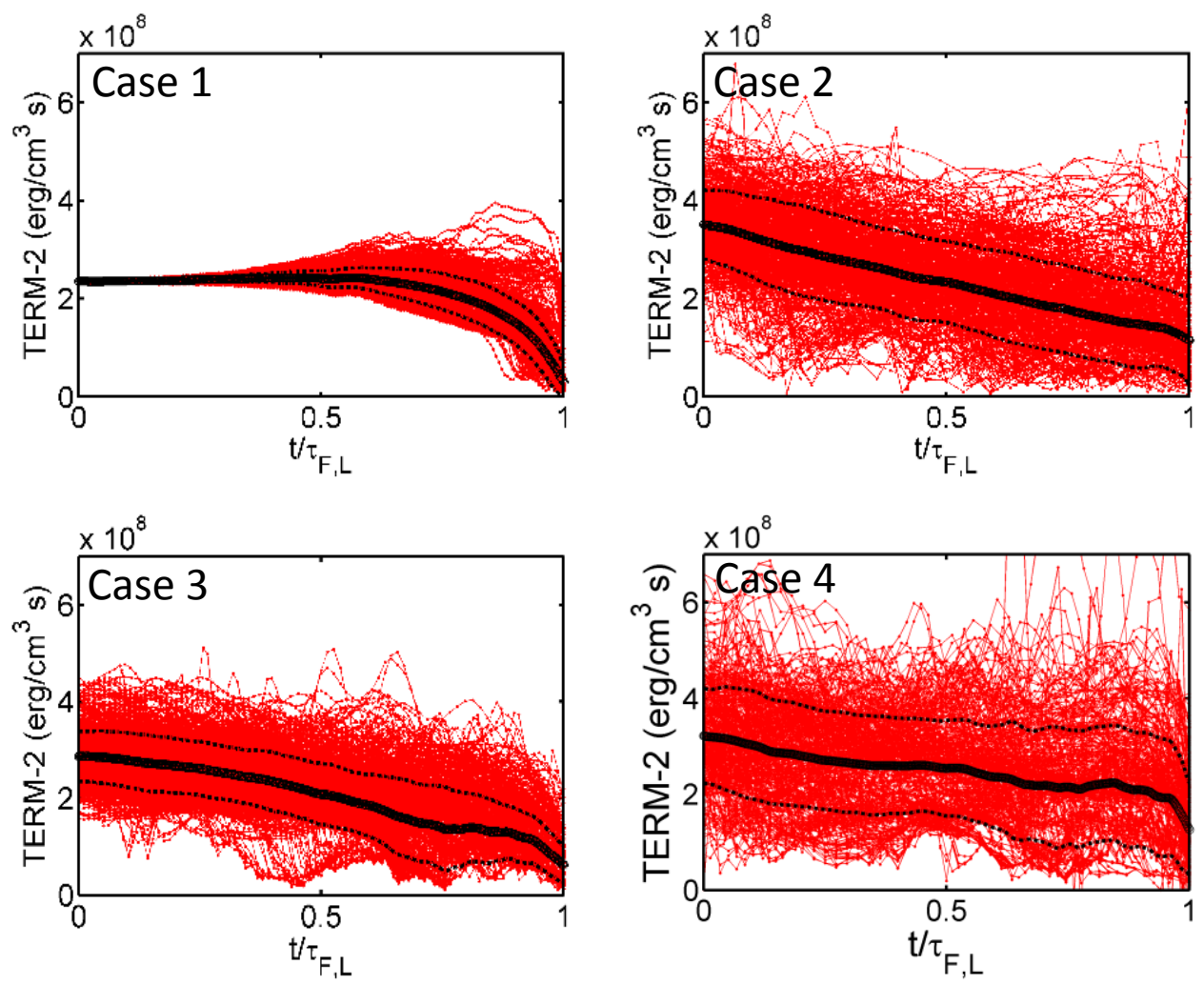

Figure 20. Term 2 of $S_{\mathrm{d}}$ equation vs. normalized time $\left(t / \tau_{F, L}\right)$ for $1321 \mathrm{~K}$ isosurface for Cases 1-4.
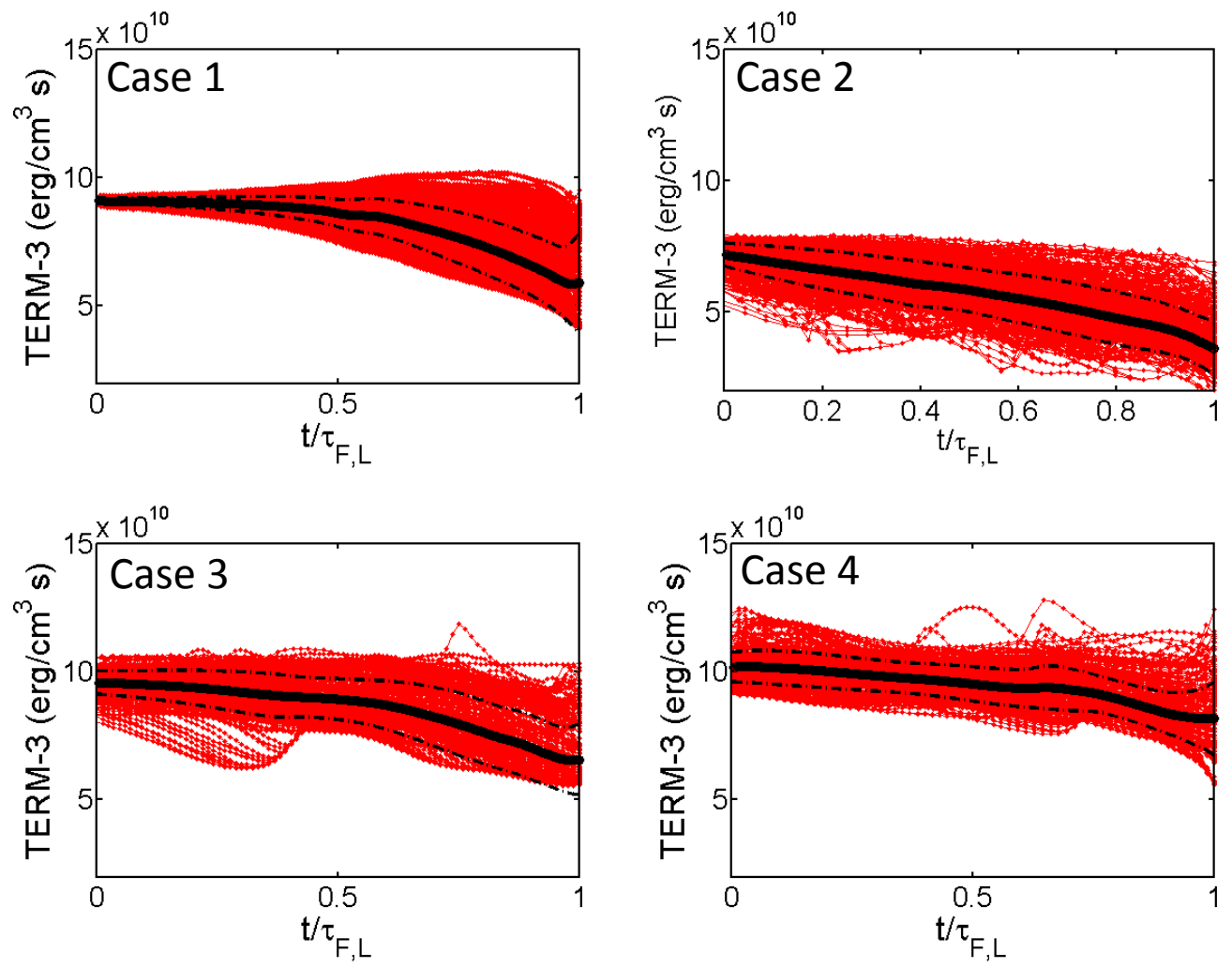

Figure 21. Term 3 of $S_{\mathrm{d}}$ equation vs. normalized time $\left(t / \tau_{F, L}\right)$ for $1321 \mathrm{~K}$ isosurface for Cases 1-4. 

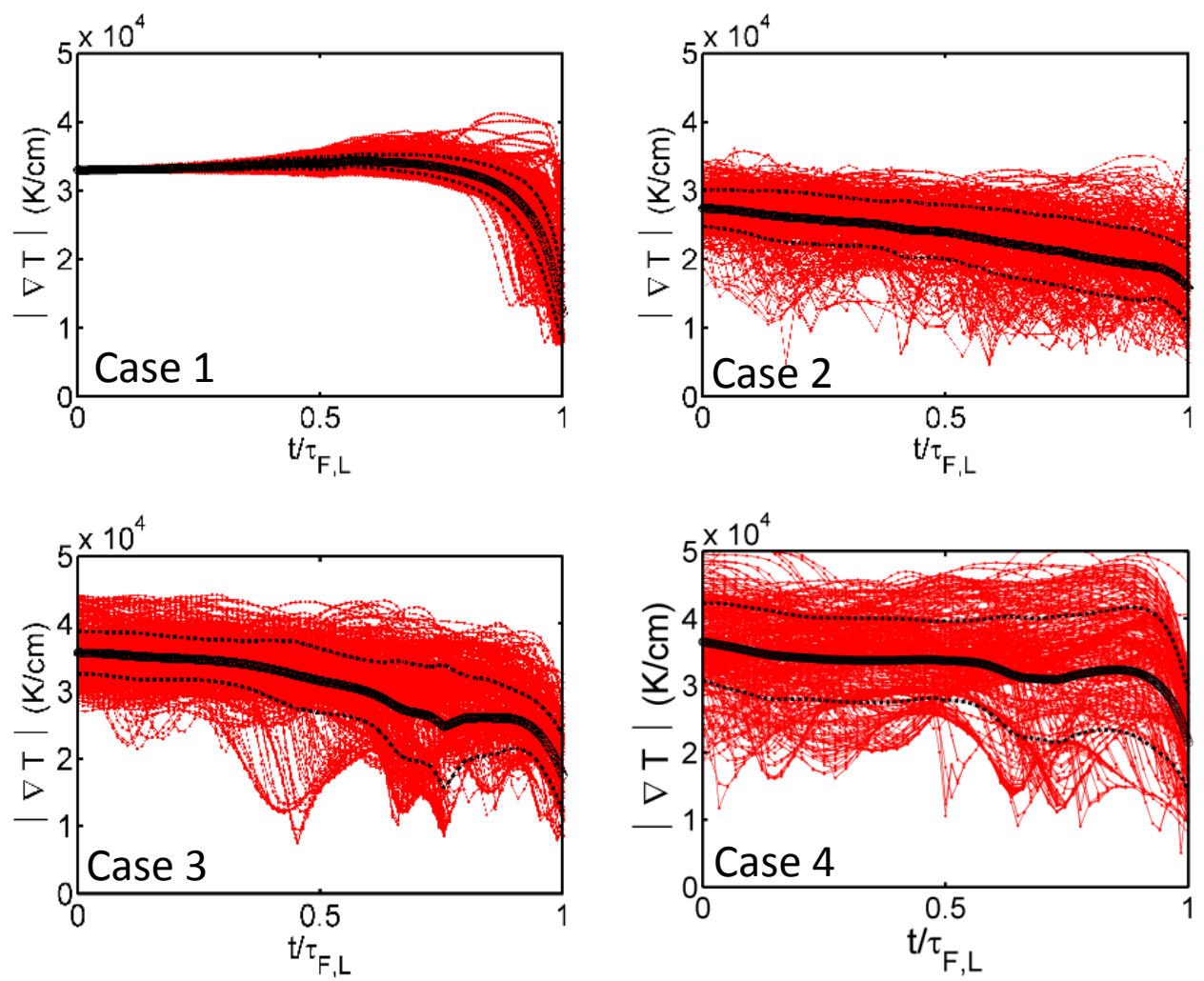

Figure 22. Magnitude of temperature gradient $(|\nabla T|)$ vs. normalized time $\left(t / \tau_{F, L}\right)$ for $1321 \mathrm{~K}$ isosurface for Cases 1-4.
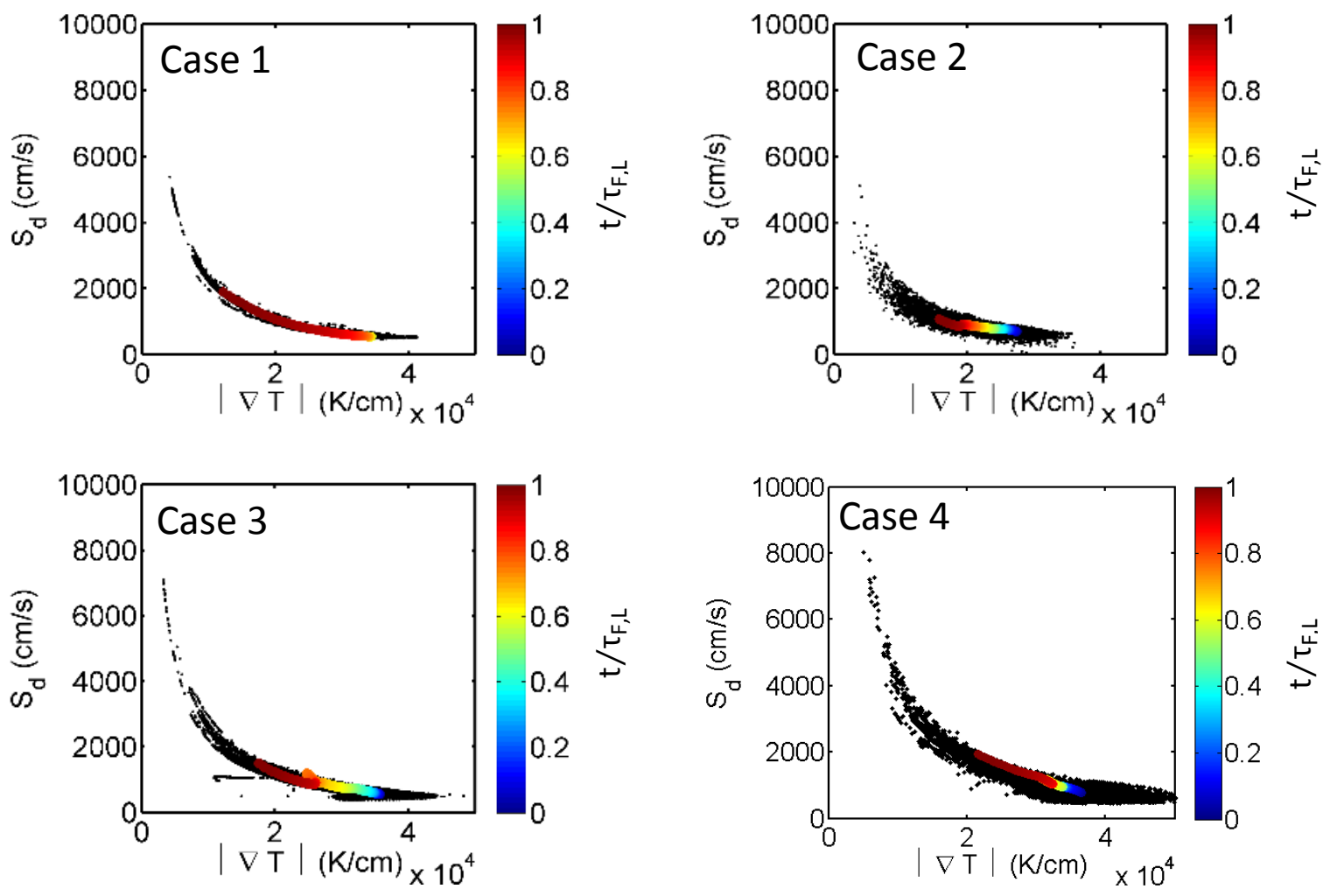

Figure 23. Joint evolution of displacement flame speed $\left(S_{\mathrm{d}}\right)$ and magnitude of temperature gradient $(|\nabla T|)$ with normalized time $\left(t / \tau_{F, L}\right)$ for $1321 \mathrm{~K}$ isosurface for Cases 1-4. 

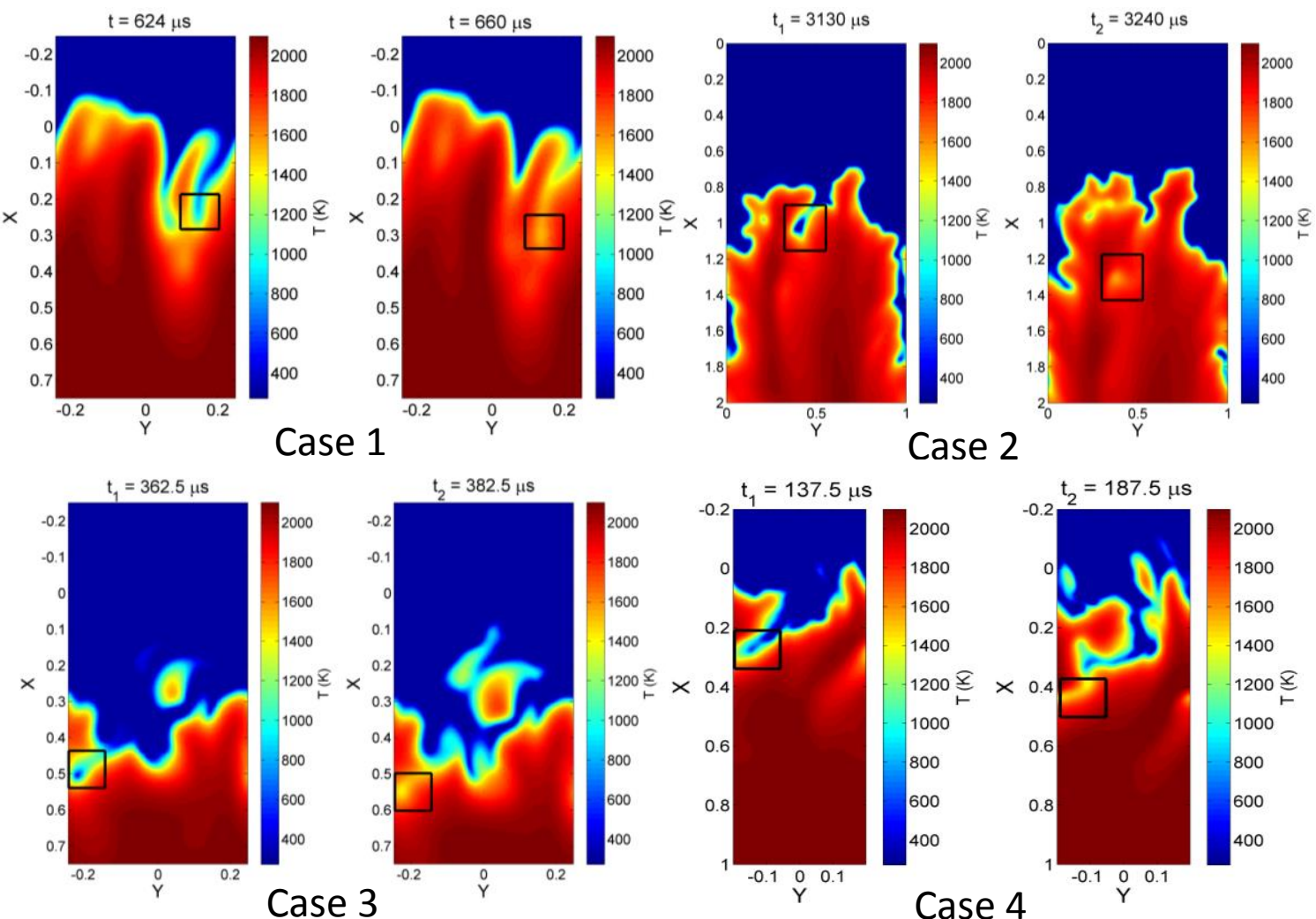

Figure 24. Volume slice of temperature $(T)$ at two time instants showing the mixing process for Cases $1-4$. 

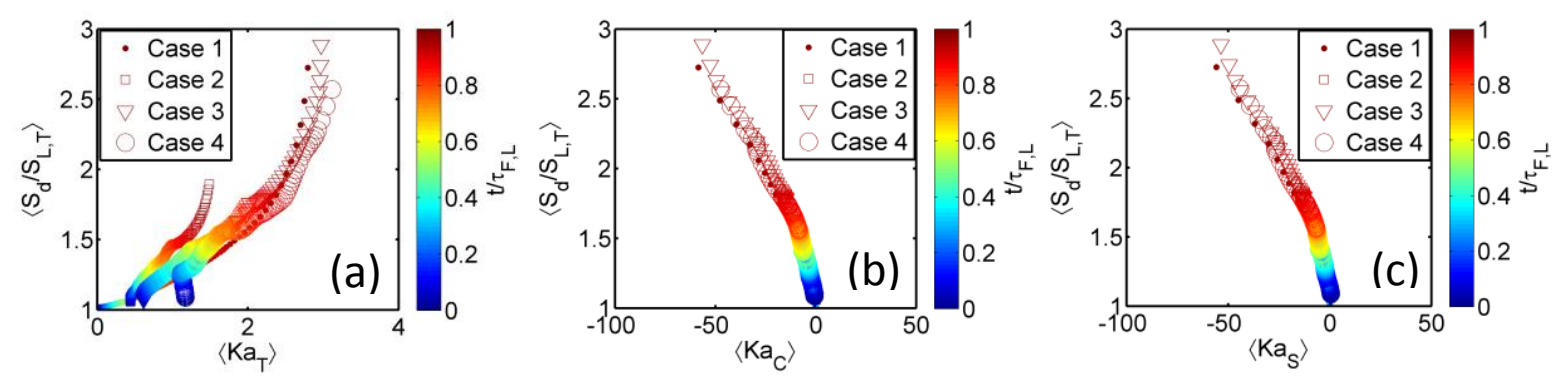

Figure 25. Time evolution of $\left\langle S_{d} / S_{L, T}\right\rangle$ versus (a) $\left\langle K a_{T}\right\rangle$, (b) $\left\langle K a_{C}\right\rangle$, and (c) $\left\langle K a_{S}\right\rangle$, for the $T=665 \mathrm{~K}$ isosurface.

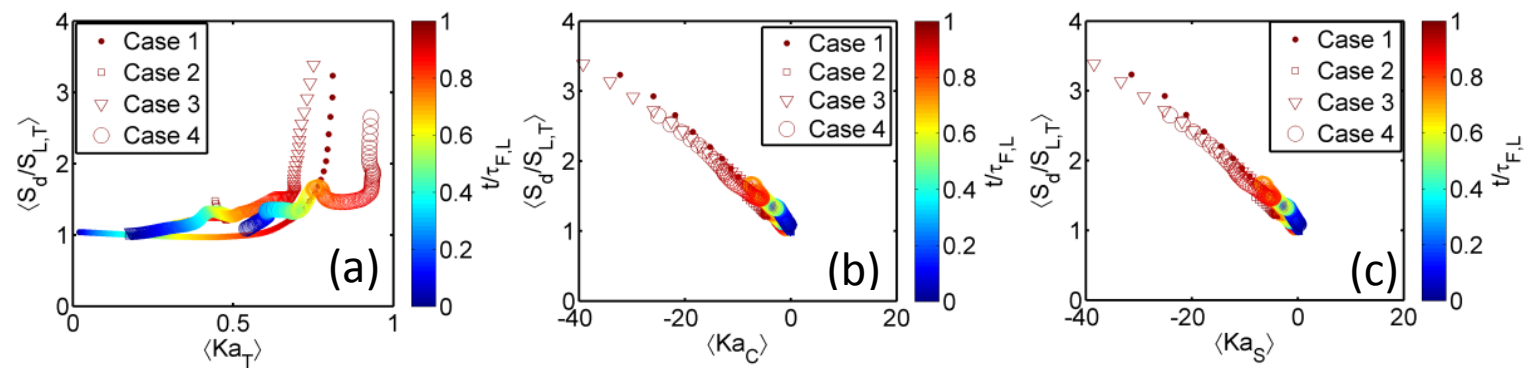

Figure 26. Time evolution of $\left\langle S_{d} / S_{L, T}\right\rangle$ versus (a) $\left\langle K a_{T}\right\rangle$, (b) $\left\langle K a_{c}\right\rangle$, and (c) $\left\langle K a_{S}\right\rangle$, for the $T=1321 \mathrm{~K}$ isosurface. 


\section{Appendix A}

The flows corresponding to Cases 1-4, approaching the flame were nearly isotropic and followed the Obukhov-Kolmogorov spectrum. The turbulent kinetic energy (TKE) spectra at the inlet face are plotted in Fig. A1. It is observed from this figure that most of the TKE spectra nearly follow $-5 / 3^{\text {rd }}$ law except for Case 1 , which was very weakly turbulent.

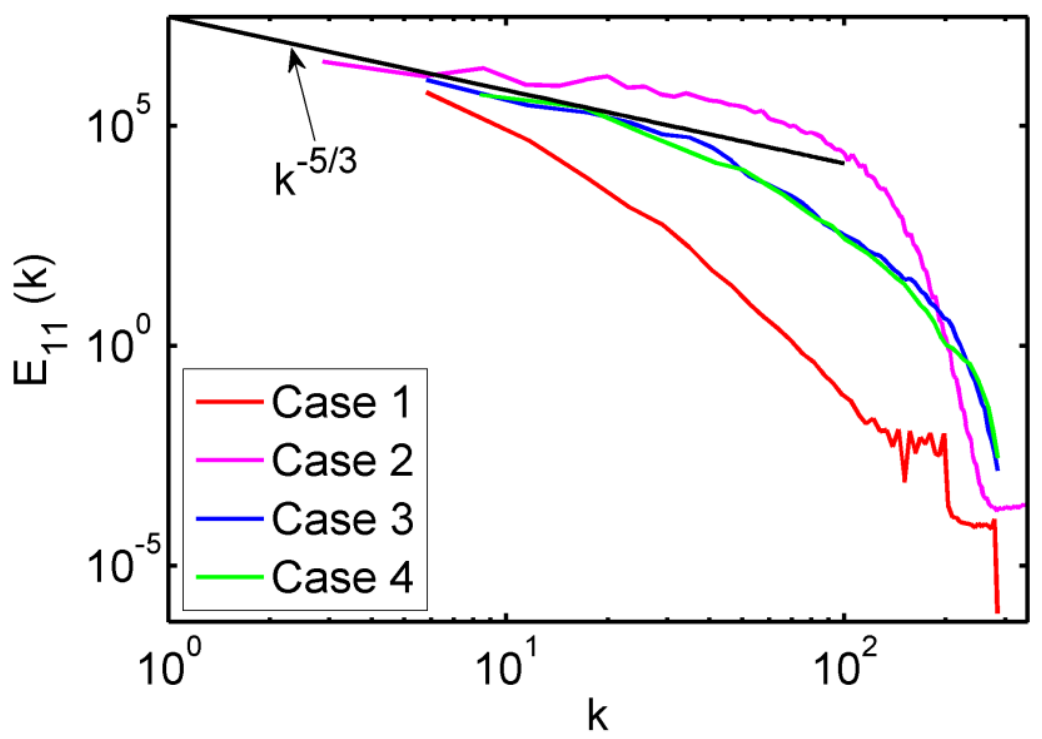

Figure A1. Turbulent kinetic energy spectrum for the four simulation cases considered. Case 1(red), Case 2 (magenta), Case 3 (blue) and Case 4 (green). The standard $-5 / 3^{\text {rd }}$ slope is shown in black. The unit of $\mathrm{k}$ is $\mathrm{cm}^{-1}$.

The JPDF of $\kappa_{1}$ and $\kappa_{2}$ for $1321 \mathrm{~K}$ isosurface obtained by sorting the principal curvatures according to their absolute magnitudes, at $t=\tau_{\text {track }}$, are shown in Fig.A2. Based on this condition, the flame surface is mostly comprised of near cylindrical geometry for Case 1 whereas hyperboloid geometries are found for Cases 2-4. 

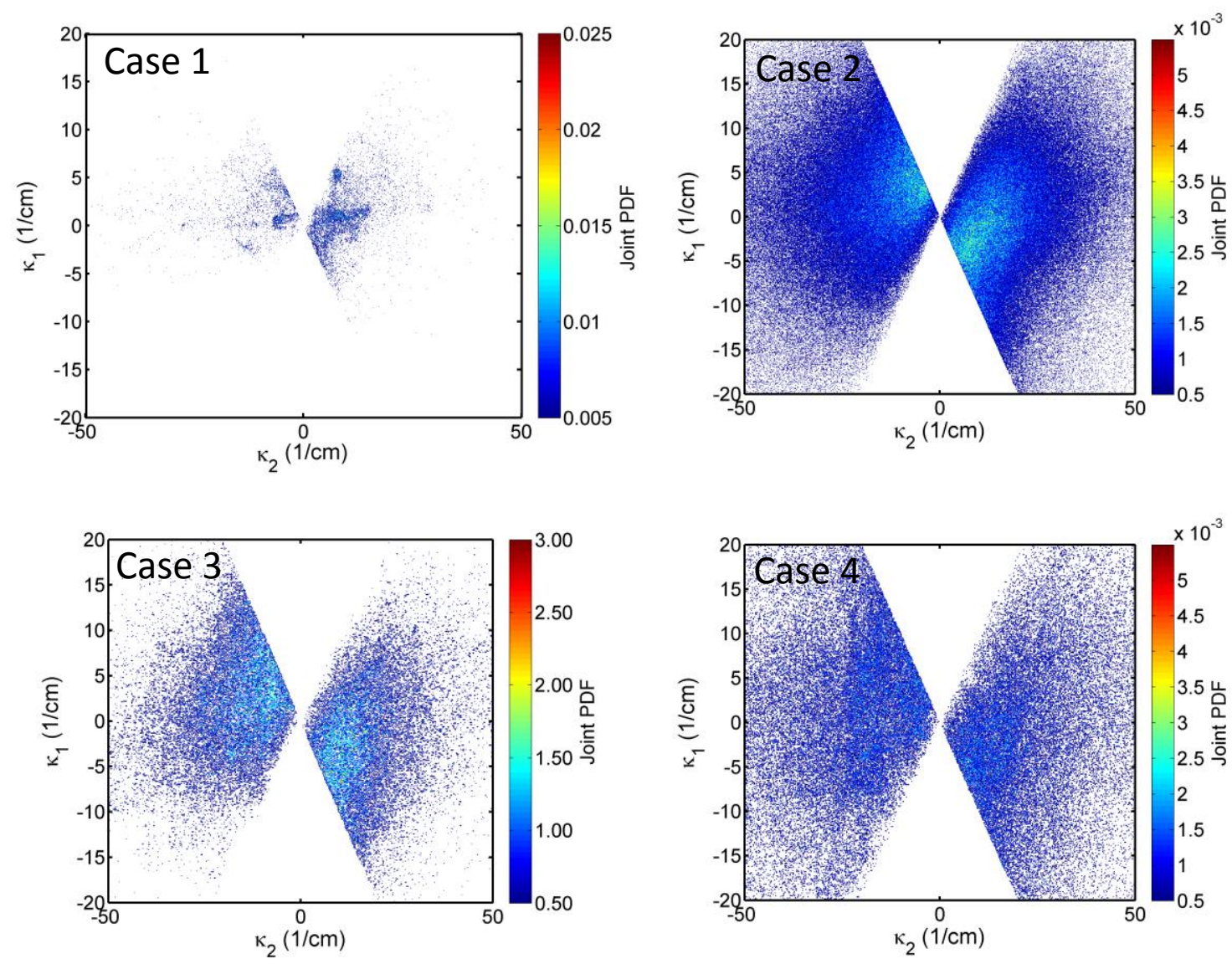

Figure A2. Joint probability density function of principal curvatures $\left(\kappa_{1}\right.$ and $\left.\kappa_{2},\left|\kappa_{1}\right|<\left|\kappa_{2}\right|\right)$ for the entire temperature isosurface of $T=1321 \mathrm{~K}$ at $t=\tau_{\text {track }}$ for Cases $1-4$. 